\title{
CHAPITRE VI
}

\section{COUPLAGES ET DYNAMIQUE}

\section{INTRODUCTION}

Au terme du chapitre $V$, la saga du Soleil à la Terre n'est pas achevée. Certes, côté jour, les photons solaires énergétiques ont créé une ionosphère et excité le gaz neutre. Nous avons étudié les effets "secondaires" de ce voyage : recombinaison chimique avec l'atmosphère, chauffage de l'ionosphère ...

Il nous reste à étudier ce qui se passe pour les particules précipitant de la magnétosphère vers l'ionosphère. Emis dans une facule ou une protubérance, un électron ou un proton s'est déplacé dans le vent solaire, a été conduit le long de la magnétopause vers le côté nuit de la Terre, en créant la cavité magnétosphérique. Au point de reconnexion, ou en ayant traversé la magnétopause vers le feuillet de plasma, il a été entraîné vers la Terre pour ensuite se diriger vers l'un des pôles magnétiques, piégé par le champ géomagnétique. En arrivant dans la thermosphère, il va ioniser et exciter l'atmosphère neutre puis, après avoir perdu la plus grande partie de son énergie, chauffer les électrons thermiques ambiants avant d'être lui-même thermalisé. Ces processus sont déjà bien connus du lecteur, puisqu'ils sont en tous points identiques aux phénomènes qui affectent les photoélectrons secondaires du chapitre $V$. Ainsi, il se crée une ionosphère aurorale et polaire, couplée fortement avec la magnétosphère, et dont nous examinerons le mécanisme et les caractéristiques.

Le couplage ionosphère/magnétosphère passe également par la fermeture des systèmes de courants définis au chapitre III. Il s'agit des conductivités ionosphériques, dont nous déterminerons les expressions. Elles nous permettront de terminer la description commencée au chapitre III des systèmes de courants qui entourent notre Terre, en abordant l'électrojet auroral et l'électrojet équatorial.

Ces deux couplages, particulaire et électromagnétique, engendrent des champs électriques aux hautes latitudes, que nous étudierons également. Ils imposent aussi une dynamique à l'ensemble thermosphère/ionosphère. L'étude de cette dynamique nous mènera au calcul du vent méridien, et nous montrera que l'ionosphère peut servir de traceur à la thermosphère. Nous en montrerons deux exemples sans les approfondir : les marées atmosphériques et les ondes de gravité déjà abordées au chapitre $I I$. 
A l'inverse des chapitres précédents, celui-ci est essentiellement basé sur des expériences, et principalement sur des expériences radar. La théorie des plasmas conduisant à la compréhension du fonctionnement des radars géophysiques est donnée en annexe III. Une présentation de ces instruments y figure également.

\section{COUPLAGE IONOSPHÈRE/MAGNÉTOSPHÈRE : LES PRÉCIPITATIONS AURORALES}

\section{CARACTÉRISTIQUES DES PRÉCIPITATIONS}

\section{a. Electrons}

Le chapitre concernant la magnétosphère a montré comment des particules issues du vent solaire traversent la magnétopause pour précipiter dans l'atmosphère terrestre en formant les ovales auroraux. Nous n'avons rien dit, jusqu'à présent, des caractéristiques de ces flux précipités, qu'on peut considérer comme la manifestation la plus directe du couplage ionosphère/magnétosphère. Deux auteurs, Hardy et Gussenhoven, aidés de collaborateurs, ont défriché ce terrain par un travail herculéen : l'analyse de millions de spectres de précipitations mesurés chaque seconde par les satellites météorologiques de la défense américaine, les satellites DMSP. Ils ont classé ces précipitations selon l'indice planétaire Kp (chapitre $I I I)$. Les détecteurs électroniques permettent une analyse de $50 \mathrm{eV}$ à $20 \mathrm{keV}$, et les détecteurs ioniques de $30 \mathrm{eV}$ à $30 \mathrm{keV}$. Les mesures sont effectuées entre 600 et $900 \mathrm{~km}$ d'altitude environ. Nous nous appuierons grandement sur leurs travaux. Concernant les précipitations électroniques, ces auteurs distinguent deux populations, selon que l'énergie moyenne est supérieure ou inférieure à $600 \mathrm{eV}$ :

- la première population électronique, d'énergie moyenne supérieure à $600 \mathrm{eV}$ est plus énergétique côté matin dans l'ovale. A Kp donné, il y a deux régions de maximum de l'énergie moyenne : après minuit et avant midi. Pour $\mathrm{Kp}=1$ par exemple, l'énergie moyenne de ces deux régions est respectivement 3,20 et $3,90 \mathrm{keV}$, tandis que les flux d'énergie correspondant sont $1,4310^{8}$ et $6,3410^{7} \mathrm{keV} \cdot \mathrm{cm}^{2} \cdot \mathrm{s}^{-1} \cdot \mathrm{sr}^{-1}$. Ces valeurs sont relativement comparables, mais la latitude du maximum nocturne $\left(66^{\circ}\right)$ est sensiblement supérieure à celle du maximum diurne $\left(70^{\circ}\right)$. Entre 12 et 18 heures, les précipitations d'électrons chauds disparaissent. Cette population constitue l'apport principal en terme de flux d'énergie de l'ovale auroral,

- la seconde population électronique, d'énergie moyenne inférieure à $600 \mathrm{eV}$, se concentre dans une région qui s'étend depuis le côté interne (le plus proche du pôle) de la région des précipitations des électrons chauds, jusqu'au pôle. Elle constitue l'apport principal, en terme de flux de particules, à haute latitude. Les flux de particules les plus importants se trouvent côté jour. Ils dessinent une région en forme de croissant, centré approximativement avant midi, et s'étendant, en temps magnétique local, sur la plus grande partie du côté diurne, avec parfois une extension nocturne. Ceci est illustré sur la figure $V I-1$. 

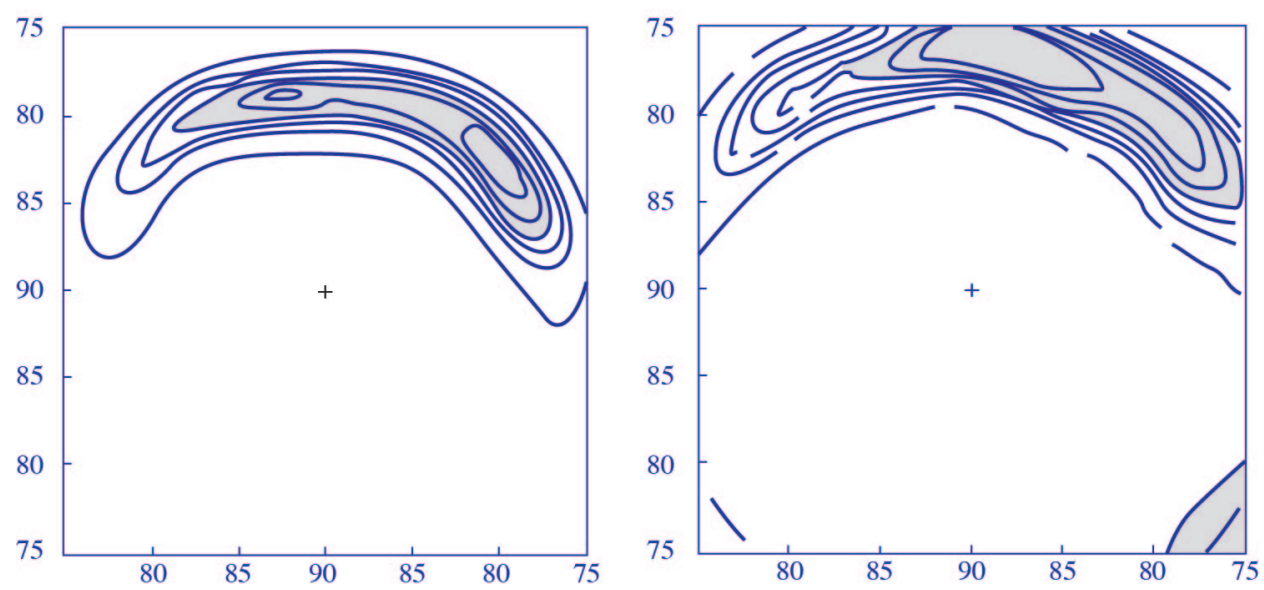

Figure VI-1 : isocontours des flux intégrés d'électrons précipités

Projections polaires géomagnétiques, midi en haut, pôle symbolisé par une croix (D.A. Hardy, M.S. Gussenhoven et E. Holeman, J. Geoph. Res., 90, 4229-4248, 1985).

Le maximum du flux de particules d'avant midi est peu sensible à l'activité magnétique. Dans ce croissant, l'énergie moyenne est comprise entre 183 et $272 \mathrm{eV}$, et le flux d'énergie est compris entre $4,8810^{7}$ et $8,4510^{7} \mathrm{keV} \cdot \mathrm{cm}^{-2} \cdot \mathrm{s}^{-1} \cdot \mathrm{sr}^{-1}$ pour $\mathrm{Kp}=1$. L'énergie moyenne possède un minimum entre 11 et 12 heures en temps magnétique local. Celui-ci est situé sur le bord interne (vers le pôle) de la région en forme de croissant.

Cette étude a conduit leurs auteurs à discerner plusieurs régions dans le cornet polaire. Ils définissent la corne comme la région autour du minimum de l'énergie moyenne, et la fente comme la région en forme de croissant. Leurs observations ont donné naissance à un modèle statistique dont les résultats sont montrés sur la figure $V I-2$ pour $\mathrm{Kp}=1$.

Cette étude est la plus complète à ce jour. Elle néglige cependant les très basses énergies. De très nombreuses mesures, tant par satellites que par fusées, ont montré que le plus souvent, les spectres énergétiques de particules précipitantes ressemblent à ceux montrés sur la figure $V I-3$.

On y distingue deux zones. La première, au-dessous de typiquement 100 à $200 \mathrm{eV}$, est très souvent décrite par une loi de puissance :

$F(E)=\alpha E^{-\gamma}$

dans laquelle $F$ représente le flux de particules et $E$ l'énergie. Les facteurs $\alpha$ et $\gamma$ sont des paramètres ajustables.

La seconde montre une bosse d'électrons dits «chauds ». Celle-ci est le plus souvent correctement modélisée par l'une des deux distributions suivantes :

Distribution maxwellienne : $\quad F(E)=\alpha_{l} \mathrm{e}^{-\frac{E-E_{\text {moxeme }}}{\gamma_{l}}}$

Distribution gaussienne :

$$
F(E)=\alpha_{2} \mathrm{e}^{-\frac{\left(E-E_{\text {moveren }}\right)^{2}}{\gamma_{2}}}
$$



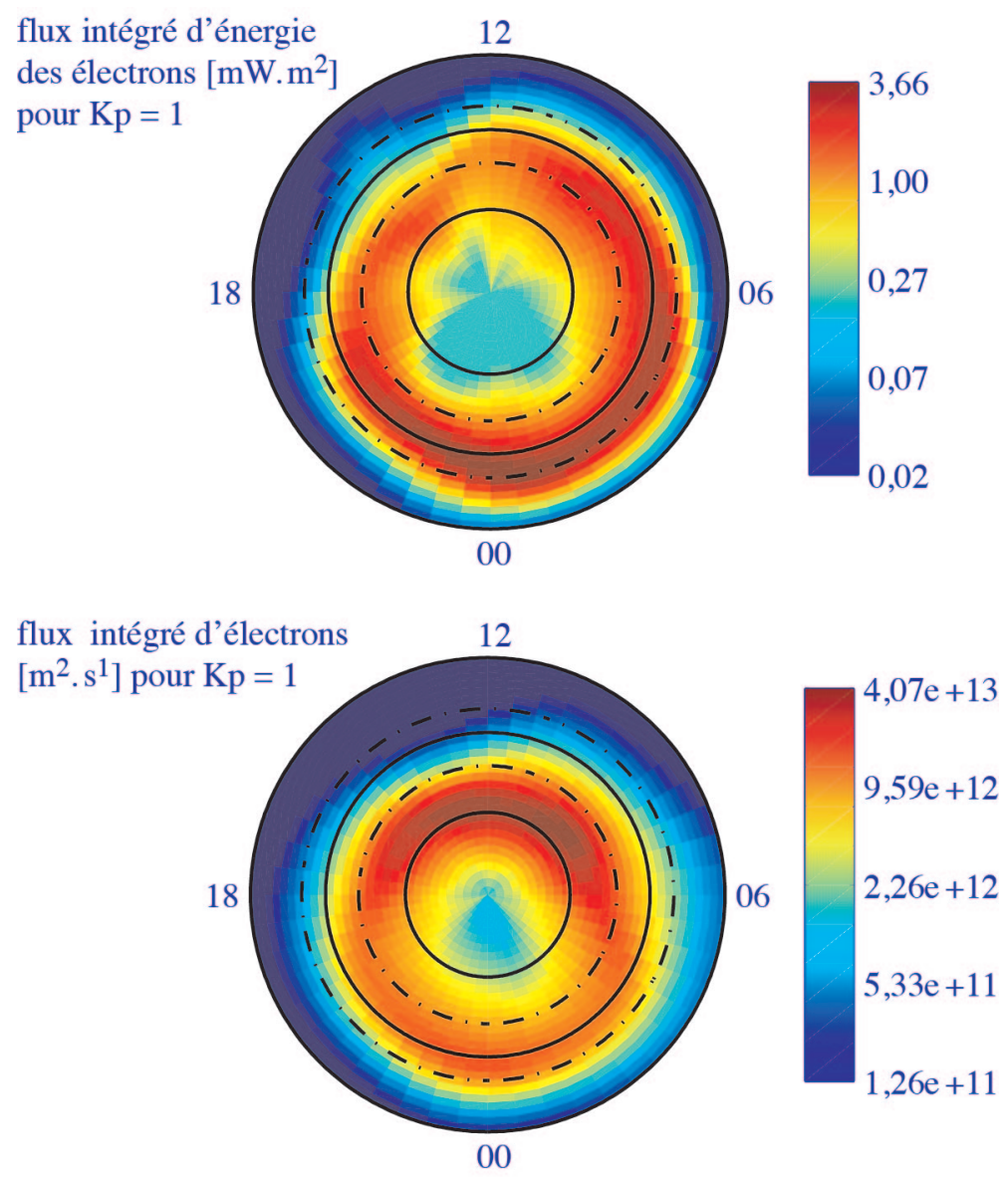

Figure $V I-2$ : cette figure montre l'ovale auroral pour $\mathrm{Kp}=1$ dans

\section{le modèle statistique basé sur les observations de Dynamics Explorer}

En haut, flux d'énergie intégré, en $\mathrm{mW} . \mathrm{m}^{-2}$. Le dessin du bas représente le flux intégré de particules $\left[\mathrm{s}^{-1} \cdot \mathrm{m}^{-2}\right]$. On retrouve les différences décrites dans le texte, en particulier sur les lieux d'intensification des flux. (D'après D.A. Hardy, M.S. Gussenhoven and E. Holeman, A statistical model of auroral electron precipitation., J. Geophys. Res., 90, 4229-4248, 1985).

Les paramètres $\alpha_{i}$ et $\gamma_{i}$ sont ici encore des paramètres ajustables. L'énergie moyenne $E_{\text {moyenne }}$ est celle du maximum de la distribution. Celle-ci est généralement de l'ordre du kilo électronvolt. L'énergie intégrée est, en général, de l'ordre du $\mathrm{mW} \mathrm{m}^{-2}$. Cependant, les précipitations peuvent être structurées en fines bandes, d'une largeur inférieure au kilomètre avec une extension spatiale qui peut atteindre plusieurs centaines de kilomètres. De telles structures s'appellent des arcs auroraux. Leur formation est encore mal comprise. De récentes mesures, jointes à de la modélisation, ont montré que dans des arcs, l'énergie intégrée peut atteindre $400 \mathrm{~mW} \mathrm{~m}^{-2}$ alors qu'un kilomètre avant ou après, elle est négligeable ! 


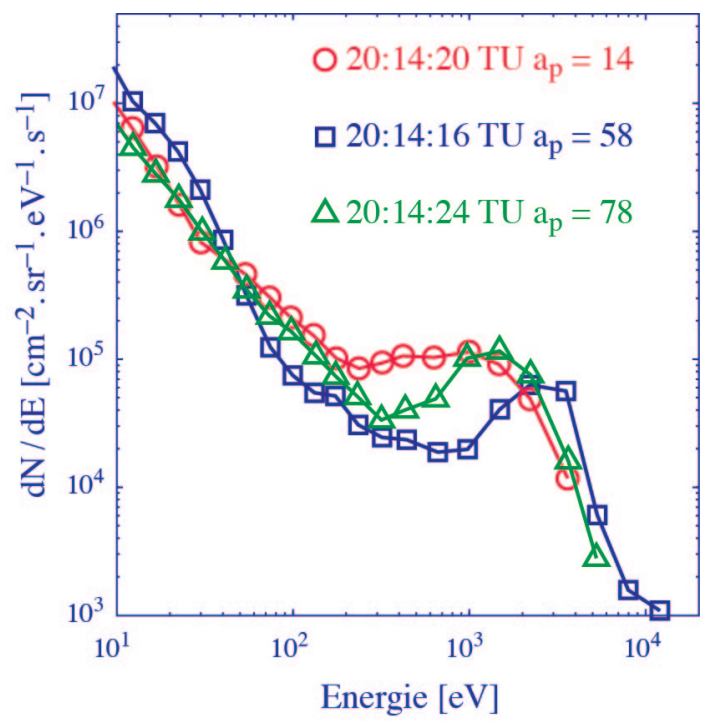

Figure VI-3 : mesure des précipitations électroniques, le 16 mai 1969

Le flux $\left[\mathrm{eV}^{-1} \mathrm{~cm}^{-2} \mathrm{~s}^{-1} \mathrm{sr}^{-1}\right]$, en ordonnée, est tracé en fonction de l'énergie [eV]. (J.D. Winningham et W.J. Heikkila, J. Geophys. Res., 79, 949, 1974).

\section{b. Protons}

Concernant les ions, les observations portent sur 26,5 millions de spectres ! Les comportements du flux intégré d'énergie, du flux de particules ou de l'énergie moyenne sont similaires d'un Kp à l'autre, d'une latitude ou d'une heure magnétique à l'autre. A Kp donné, les flux intégrés d'énergie les plus intenses forment une région en forme de $\mathrm{C}$, symétrique autour de l'axe méridien et s'étendant d'avant midi à avant minuit. Le maximum du flux intégré d'énergie se situe avant minuit, et le minimum avant midi. Avant minuit, pour $\mathrm{Kp}=1$, l'énergie moyenne est de $13,6 \mathrm{keV}$ et le flux d'énergie de $5,5210^{7} \mathrm{keV} \mathrm{cm}^{-2} \mathrm{~s}^{-1} \mathrm{sr}^{-1}$. Les mêmes grandeurs, sont, pour le minimum diurne respectivement $2,54 \mathrm{keV}$ et le flux d'énergie de $1,2310^{7} \mathrm{keV} \mathrm{cm}^{-2} \mathrm{~s}^{-1} \mathrm{sr}^{-1}$. En dehors de la région d'avant midi, le flux d'énergie intégré croît avec Kp. Le maximum de ce flux croît même d'un facteur 6,1 lorsque $\mathrm{Kp}$ va de 0 à 6 . Le flux de particules intégré est centré, lui, à midi. Il définit le centre de la corne polaire. Sa valeur est à peu près constante avec Kp. La position de son maximum a tendance à aller vers des latitudes plus basses lorsque Kp augmente. Il existe un minimum de l'énergie moyenne avant midi, mais plusieurs degrés plus au nord que celui du flux d'énergie intégré. La distance entre les deux maxima croît avec Kp. Enfin, l'énergie moyenne est maximum côté après-midi de l'ovale, près du bord équatorial des précipitations ioniques, et se décale vers midi lorsque Kp augmente.

\section{c. Comparaison}

Ces observations ne seraient pas complètes sans une comparaison des caractéristiques des précipitations ioniques et électroniques. Cette comparaison montre que le flux d'ions 
intégré moyen est une à deux fois plus petit que celui des électrons, quelles que soient la latitude, l'heure ou l'activité magnétique. Le flux d'énergie intégré des ions, côté aprèsmidi de l'ovale, peut égaler ou excéder celui des électrons près du côté équatorial de la région aurorale. Enfin, l'intégration globale hémisphérique des flux de particules donne un rapport ion sur électron variant de 0,024 à 0,9015 pour Kp augmentant de 0 à 6 -. Le rapport des flux d'énergie ne montre pas de lien avec l'activité, et est de l'ordre de 0,14. Les nombres exacts se trouvent dans le tableau $V I-1$ :

\section{Tableau VI-1 : Comparaison des flux de particules $\left[\mathrm{s}^{-1} \mathrm{sr}^{-1}\right]$ et des flux d'énergie [W]} sur tout l'hémisphère pour les ions et les électrons

(D.A. Hardy, M.S. Gussenhoven and E. Holeman, A statistical model of auroral ion precipitation, J. Geophys. Res., 94, 370-392, 1989)

\begin{tabular}{lcccc}
\hline $\boldsymbol{K}_{\boldsymbol{p}}$ & $\begin{array}{c}\text { Flux } \\
\boldsymbol{d}^{\prime} \text { ions }\end{array}$ & $\begin{array}{c}\text { Flux } \\
\boldsymbol{d}^{\prime} \text { électrons }\end{array}$ & $\begin{array}{c}\text { Flux d'énergie } \\
\text { des ions }\end{array}$ & $\begin{array}{c}\text { Flux d'énergie } \\
\text { des électrons }\end{array}$ \\
\hline $\boldsymbol{0}$ & $3,6210^{23}$ & $1,5310^{25}$ & $7,2610^{8}$ & $4,6810^{9}$ \\
$\mathbf{1}$ & $3,8010^{23}$ & $1,8510^{25}$ & $1,3810^{9}$ & $8,1410^{9}$ \\
$\mathbf{2}$ & $4,5410^{23}$ & $2,3510^{25}$ & $2,0310^{9}$ & $1,4910^{10}$ \\
$\mathbf{3}$ & $5,5310^{23}$ & $3,0810^{25}$ & $2,9210^{9}$ & $2,5910^{10}$ \\
$\mathbf{4}$ & $7,1010^{23}$ & $4,0510^{25}$ & $4,1010^{9}$ & $3,3610^{10}$ \\
$\mathbf{5}$ & $8,7410^{23}$ & $5,8310^{25}$ & $5,4710^{9}$ & $4,3210^{10}$ \\
$>\mathbf{6 -}$ & $1,5010^{24}$ & $1,0210^{26}$ & $1,0010^{10}$ & $7,7610^{10}$ \\
\hline
\end{tabular}

L'observation des précipitations de protons n'est pas récente : dès 1939, Vegard avait détecté dans les spectres auroraux de larges raies décalées de quelques Angströms par rapport aux raies $\mathrm{H}$ de la série de Balmer. Il avait alors supposé que ces raies étaient dues à des précipitations d'hydrogène provenant du soleil, et que les décalages étaient liés à des erreurs de mesure. Ces observations furent attribuées à l'effet Doppler. En effet, les protons, provenant de la magnétosphère et précipités dans l'atmosphère terrestre le long des lignes de force du champ géomagnétique, subissent des réactions d'échange de charge conduisant à la formation d'atomes d'hydrogène ; ceux-ci, à leur tour, réagissent avec les neutres ambiants. Certaines de ces collisions conduisent à l'excitation d'atomes d'hydrogène. Ces atomes sont produits dans des états excités et sont en quantité suffisante pour que des émissions $\mathrm{H}$, en particulier certaines raies de la série de Balmer $(\mathrm{H} \alpha=656,3 \mathrm{~nm}$ et $\mathrm{H} \beta=486,1 \mathrm{~nm})$, soient détectées depuis le sol. Les atomes d'hydrogène émettent aussi des raies de la série Lyman $(\mathrm{L} \alpha=121,6 \mathrm{~nm})$. Celles-ci sont situées en dehors du spectre visible. Elles sont absorbées dans l'atmosphère ; elles ne sont donc pas détectables au sol, mais uniquement à partir d'un satellite.

La majorité des émissions $\mathrm{H} \alpha$ et $\mathrm{H} \beta$ est issue d'une région située entre 90 et $150 \mathrm{~km}$ d'altitude, le maximum d'intensité étant situé vers $110-120 \mathrm{~km}$. Le profil d'intensité dépend 
toutefois de l'importance des précipitations et de l'énergie initiale des particules incidentes. Par conséquent, les émissions $\mathrm{H} \alpha$ et $\mathrm{H} \beta$ produites par les atomes d'hydrogène excités constituent une véritable signature propre aux précipitations de protons.

La redistribution angulaire des protons et atomes d'hydrogène possède plusieurs origines. La première est le phénomène de miroir magnétique, discuté au chapitre sur la magnétosphère : les lignes de forces du champ géomagnétique se resserrent lorsque l'altitude diminue, ce qui, par conservation de l'énergie et du premier invariant adiabatique, conduit en un transfert d'énergie le long de l'axe du champ vers l'axe perpendiculaire. Les collisions constituent la seconde cause de redistribution angulaire.

\section{d. Précipitations des latitudes moyennes et basses}

Si les précipitations de particules sont intenses dans les zones aurorales, elles n'y sont pas cantonnées. En dessous de $58^{\circ}$ de latitude, l'ionosphère est en effet soumise à la plasmasphère. Les précipitations de particules y ont un flux d'énergie au moins mille fois inférieur à celui des hautes latitudes, mais celui-ci contribue cependant à créer une ionosphère nocturne, y compris au-dessus de l'équateur ou des tropiques. La structure latitudinale de ces précipitations montre que les ions y ont un maximum vers $52^{\circ}$, sont négligeables entre $48^{\circ}$ et $20^{\circ}$, et ont de nouveau un maximum au-dessus de l'équateur. Ces deux maxima ont une amplitude en terme de flux d'énergie $10^{-3}$ fois celle du maximum auroral, vers $65^{\circ}$.

Les électrons connaissent eux aussi un maximum vers $52^{\circ}$ et un second situé vers $27^{\circ}$. Leurs amplitudes sont plus proche de $10^{-4}$ fois celle du maximum auroral, vers $70^{\circ}$.

\section{EFFET DES PRÉCIPITATIONS DE PARTICULES : L'IONOSPHÈRE NOCTURNE}

Le mécanisme de précipitations s'apparente à celui de la photoionisation à ceci près que l'on remplace les photons par des électrons ou des protons. Ainsi, on peut avoir, comme pour la photoionisation, une approche simplifiée et une approche cinétique. La première considère qu'il faut environ $\Delta \varepsilon=35 \mathrm{eV}$ pour ioniser un constituant neutre. Cette ionisation résulte d'un choc entre les particules énergétiques et ce constituant; au cours du choc, la particule incidente cède $\Delta \varepsilon$. En conséquence, si $E$ est l'énergie initiale de la particule, elle est susceptible de produire $\frac{E}{\Delta \varepsilon}$ ionisations. On peut supposer que la section efficace offerte par le constituant neutre est proportionnelle à sa masse. Considérons une colonne cylindrique de diamètre unitaire, remplie du constituant neutre à une concentration uniforme dans le volume, et une particule énergétique qui arrive au sommet du cylindre suivant son axe, pénètre dans le milieu et ionise tout au long de sa trajectoire, perdant $\Delta \varepsilon$ à chaque ionisation, jusqu'à atteindre une énergie inférieure à $\Delta \varepsilon$, limite en deçà de laquelle son pouvoir ionisant disparaît. Si on mesure la masse de gaz au travers de laquelle la particule a pénétré jusqu'à perdre son pouvoir ionisant, on obtient une grandeur qui quantifie le pouvoir de pénétration de la particule et que l'on appelle profondeur de pénétration: cette grandeur 
dépend de l'énergie initiale. Différentes expériences menées avec des électrons ont conduit à l'ajustement suivant de cette profondeur de pénétration $R(E)$ :

$R(E)=4,310^{-6}+5,3610^{-5} E^{1,67}$

où $R(E)$ est la profondeur exprimée en $\mathrm{kg} \mathrm{m}^{-2}$ et $E$ l'énergie en $\mathrm{keV}$. On considère que cette expression est valable entre $200 \mathrm{eV}$ et $50 \mathrm{keV}$. Lorsque la particule incidente ionise, elle subit une déviation par rapport à sa trajectoire initiale. De fait, l'efficacité de l'ionisation n'est pas uniforme le long de la trajectoire. Si on note s la profondeur de diffusion, qui représente la masse rencontrée jusqu'au point où l'on se trouve, on introduit une fonction de dissipation d'énergie $\Lambda\left(\frac{s}{R(E)}\right)$ qui quantifie la répartition de l'ionisation entre le point d'entrée et le point d'arrêt $R(E)$. La condition :

$\int_{-1}^{1} \Lambda\left(\frac{s}{R(E)}\right) d\left(\frac{s}{R(E)}\right)=1$

assure la conservation de l'énergie des particules incidentes. La borne -1 dans l'intégrale précédente signifie que des particules peuvent rebrousser chemin à la suite de collisions dans le milieu, et la borne +1 correspond à une diffusion en avant. Dans l'atmosphère, si on note $\rho(z)$ la masse volumique à l'altitude $z$, on a comme expression de $s$ :

$s=\int_{z}^{\infty} \rho(z) d z$

$\Lambda\left(\frac{s}{R(E)}\right) \frac{\rho(z)}{R(E)}$ représente alors la proportion de chocs qui se produisent à l'altitude $z$. Si l'on considère un flux monoénergétique de particules d'énergie $E$ et d'intensité $F$, le produit $F \frac{E}{\Delta \varepsilon}$ représente le nombre total d'ionisations qui sont possibles avec ce faisceau, et on obtient comme expression du taux de production ionique :

$P(z, E)=F \frac{E}{\Delta \varepsilon} \Lambda\left(\frac{s}{R(E)}\right) \frac{\rho(z)}{R(E)}$

L'énergie déposée à l'altitude $z$ est alors :

$\varepsilon(z, E)=\Delta \varepsilon P(z, E)$ $V I-8$

$\mathrm{Si}$ on a un spectre en énergie de particules précipitantes, au lieu d'un faisceau monoénergétique, la production devient :

$P(z)=\int_{0}^{\infty} \frac{E}{\Delta \varepsilon} \Lambda\left(\frac{s}{R(E)}\right) \frac{\rho(z)}{R(E)} F(E) d E$

où $F(E)$ représente la densité spectrale de flux à l'énergie $E$. 
La figure VI-4 donne le taux de production en fonction de l'altitude pour différentes énergies. La fonction de dissipation joue un rôle très important autour du maximum de production et c'est elle qui conditionne principalement la forme de la production autour du maximum. On peut considérer que le maximum de production est atteint à l'altitude à laquelle $s=R(E)$, car la fonction $\Lambda$ ne modifie que très peu la position du maximum.

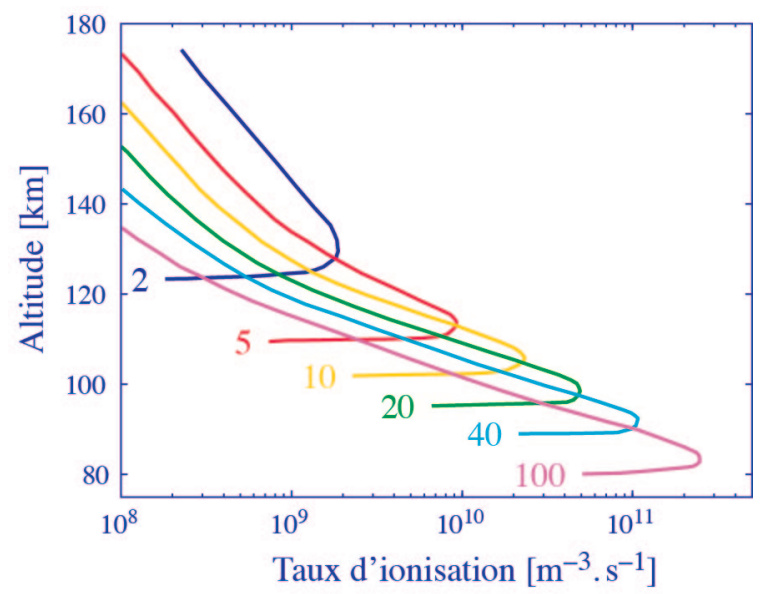

Figure VI-4 : profils verticaux des taux d'ionisations normalisés pour différents flux d'électrons monoénergétiques précipitants dans l'atmosphère le long des lignes de champ magnétique

Les énergies précipitantes sont indiquées auprès de chaque courbe (en keV).

On constate que lorsque l'énergie croît, l'altitude du maximum de production décroît $(R(E)$ croît) et la valeur de ce maximum croît $\left(\frac{E}{\Delta \varepsilon}\right.$ croît). Alors que le maximum est à $130 \mathrm{~km}$ pour une énergie de $2 \mathrm{keV}$, il est à $100 \mathrm{~km}$ pour une énergie de $20 \mathrm{keV}$ et à $80 \mathrm{~km}$ pour une énergie de $100 \mathrm{keV}$. Cette faible variation de l'altitude de pénétration des particules incidentes est due à la forte augmentation de la masse volumique lorsque l'altitude décroît. Audessus du maximum de production $\Lambda\left(\frac{S}{R(E)}\right)$ varie peu et la production décroît comme la concentration en constituant neutre, au travers de $\rho(z)$, de manière similaire à ce que l'on observe dans la photoionisation.

Malheureusement, comme dans le cas de la photoionisation, cette approche est trop simple pour décrire parfaitement les phénomènes ionosphériques. La répartition à $35 \mathrm{eV}$ par ionisation ne représente qu'une moyenne. En effet, les électrons secondaires, créés par l'impact d'une particule (photon, électron ou ion) sont susceptibles à leur tour d'aller ioniser un constituant neutre, si leur énergie cinétique acquise dans l'ionisation est suffisante. Ce mécanisme de production secondaire d'électrons représente environ un tiers de la production primaire d'électrons, mais peut devenir en région $E$, supérieure à la production primaire. Une description détaillée passe par la résolution de l'équation cinétique de transport de Boltzmann. Il s'agit de la même physique que celle développée dans le chapitre $V$ 
(équation $V-50$ ). La source est cependant à présent constituée du flux des électrons ou protons précipités étudiés ci-dessus.

La figure VI-5 montre les résultats d'une approche cinétique pour l'étude de l'ionosphère dans l'ovale auroral (en haut) et dans la calotte polaire (en bas). Les flux incidents au sommet de l'ionosphère sont soit des flux d'électrons seuls (à gauche) ou des flux d'électrons et protons (à droite). Ce sont ceux présentés ci-dessus, issus de l'étude statistique de Hardy et coauteurs. Les heures, en abscisse, sont en temps universel. Les résultats ne sont pas seulement fonction de l'intensité des précipitations, mais aussi de la composition de l'atmosphère neutre. Dans l'ovale auroral, le trou de production est bien visible autour de midi. Il est moins fort cependant lorsque les protons s'ajoutent aux électrons, puisque les précipitations de protons ont un maximum secondaire autour de cette période. Il en va de même dans la calotte polaire, où les électrons ont un minimum vers 10 heures. Autour de cette période, ce sont les protons qui créent toute l'ionisation. Tout le long de la journée, les protons sont responsables de la production au-dessous de $160 \mathrm{~km}$ dans la calotte polaire.

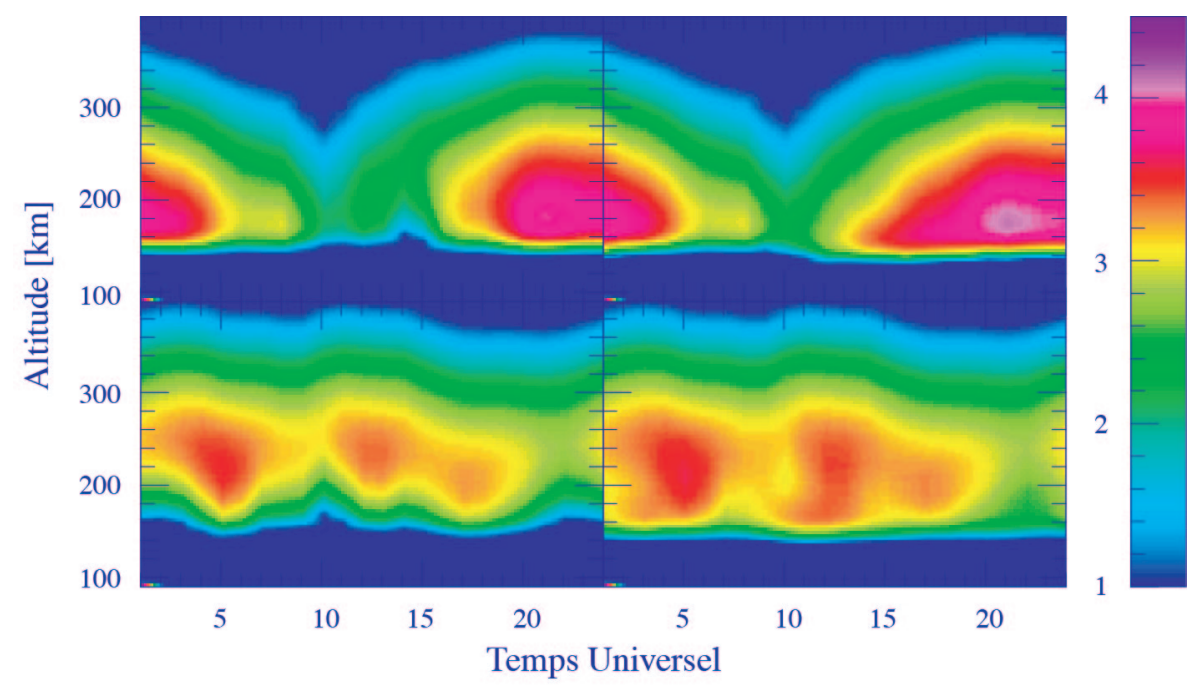

Figure VI-5 : production électronique en échelle logarithmique [électrons. $\mathrm{cm}^{-3} \cdot \mathrm{s}^{-1}$ ]

A gauche, en ne considérant que des précipitations électroniques. A droite, en considérant des précipitations d'électrons et d'ions. En haut, à $62^{\circ}$ de latitude nord, dans l'ovale auroral. En bas, à $80^{\circ}$ de latitude nord, dans la calotte polaire.

Ces résultats se retrouvent sur la figure $V I-6$, qui montrent les densités électroniques correspondantes. 


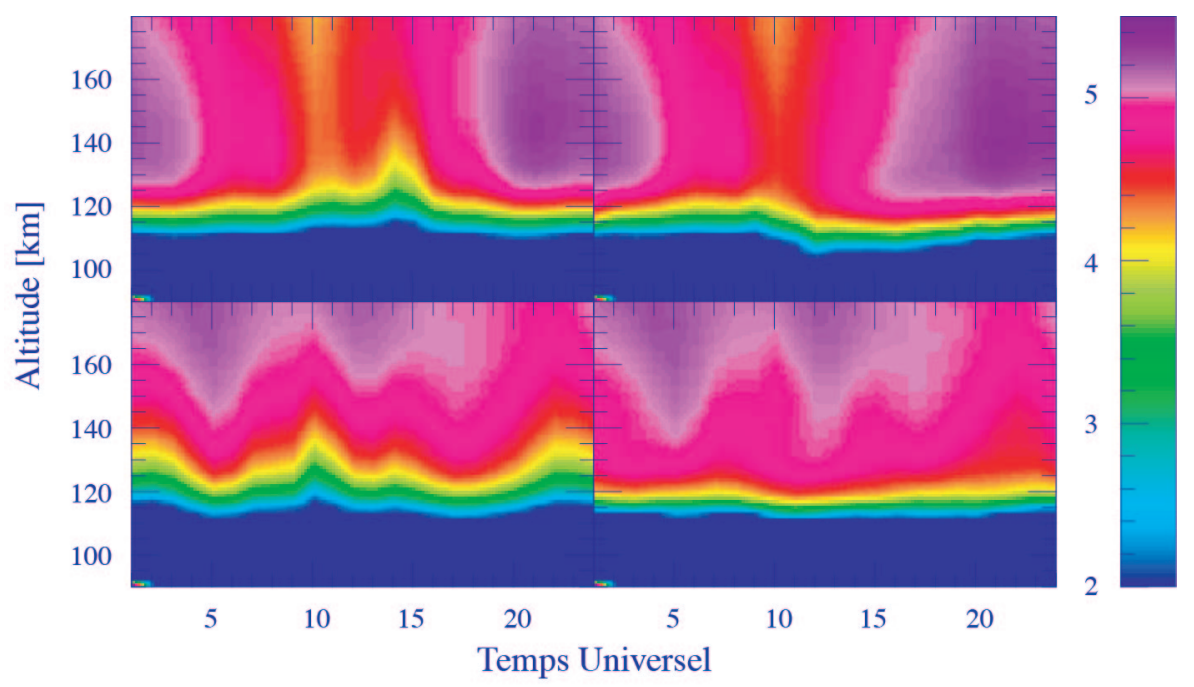

Figure VI-6 : densité électronique en échelle logarithmique [électrons . $\mathrm{cm}^{-3}$ ]

A gauche, en ne considérant que des précipitations électroniques. A droite, en considérant des précipitations d'électrons et d'ions. En haut, à $62^{\circ}$ de latitude nord, dans l'ovale auroral. En bas, à $80^{\circ}$ de latitude nord, dans la calotte polaire

\section{COUPLAGE IONOSPHÈRE/THERMOSPHÈRE / MAGNÉTOSPHÈRE}

\section{CONDUCTIVITÉ ET ÉLECTROJETS}

Au chapitre III, nous avons étudié le mouvement individuel de particules dans des champs électrique et magnétiques en résolvant le principe fondamental de la dynamique.

Cette approche se justifiait alors par les faibles concentrations du plasma rencontrées dans ces régions et donc leur caractère non collisionnel et individuel prépondérant. Au niveau de l'ionosphère, le problème est sensiblement différent puisque les concentration élevées que l'on rencontre, rendent le milieu collisionnel et les effets "collectifs" obligent donc à aborder la question avec une approche magnétohydrodynamique. Si nous considérons une population du plasma, de masse $m$ et de charge $q$, de concentration $n$, de vitesse $\vec{u}$ et de pression $p$, soumise à l'action des forces due à la gravité $\vec{g}$ et aux champ électrique $\vec{E}$ et magnétique $\vec{B}$, l'équivalent "fluide" du principe fondamental de la dynamique est l'équation de conservation de la quantité de mouvement, qui s'écrit (voir équations $A I-83$ et $A I-89$ ) :

$n m\left(\frac{\partial \vec{u}}{\partial t}+(\vec{u} \cdot \vec{\nabla}) \vec{u}\right)+\vec{\nabla} p-n m \vec{g}-n q(\vec{E}+\vec{u} \wedge \vec{B})=\frac{\delta \vec{M}}{\delta t}$

En utilisant une approche simplifiée et en considérant que les autres constituants du plasma forment un fond immobile, on peut écrire : 
$\frac{\delta \vec{M}}{\delta t}=-v n m \vec{u}$

où $v$ est la somme des fréquences de collisions de l'espèce considérée avec les populations constituant le fond. Au niveau de l'ionosphère, et plus précisément en région $E$ où se développe le mécanisme que nous voulons mettre en évidence, $v$ est de l'ordre de $10^{3} \mathrm{~s}^{-1}$ (voir chapitre $V$ ) soit des temps caractéristiques d'évolution vers l'équilibre très petit devant les échelles de temps auxquelles on s'intéresse (la seconde ou plus). Par conséquent, l'équation VI-10 peut être simplifiée et écrite à l'état stationnaire. Par ailleurs, les vitesses atteintes sont relativement faibles (du fait des collisions) pour pouvoir négliger la contribution due à la dérivée particulaire $(\vec{u} \cdot \vec{\nabla}) \vec{u}$. De fait on aboutit à l'équation de transport suivante :

$\vee m \vec{u}=-\frac{1}{n} \vec{\nabla} p+m \vec{g}+q(\vec{E}+\vec{u} \wedge \vec{B})$

La force d'origine magnétique joue un rôle particulier et nous la dissocierons donc des autres forces que nous regrouperons sous le dénominatif commun $\vec{F}$, défini par :

$\vec{F}=-\frac{1}{n} \vec{\nabla} p+m \vec{g}+q \vec{E}$ $V I-13$

L'équation du mouvement s'écrit alors :

$v m \vec{u}+q \vec{B} \wedge \vec{u}=\vec{F}$ VI-14

L'effet que nous étudions se situe principalement dans un plan perpendiculaire à l'axe défini par le champ magnétique. La gravité apporte une contribution mineure à la force $\vec{F}$. Par ailleurs, les gradients de pression horizontaux sont relativement faibles (les échelles horizontales peuvent atteindre quelques centaines de kilomètres) et nous pouvons donc considérer que la contribution majeure à $\vec{F}$ est la force électrique que nous décomposerons en une composante parallèle au champ magnétique, qui définit l'axe parallèle noté //, et en une composante perpendiculaire au champ, qui définit ainsi l'axe $P$ (pour Pedersen). L'axe $H$ (pour Hall) perpendiculaire aux deux directions précédentes permet de définir un trièdre orthonormé direct sur lequel on peut projeter les équations. Pour cela, nous introduisons le tenseur d'ordre 2 (voir annexe $I$ ) $\underline{\underline{\mu}}$ dont la représentation matricielle de l'inverse (i.e. $\underline{\underline{\mu}}^{-l}$ ) dans la base définie précédemment est :

$\underline{\underline{\mu}}^{-1}=m\left(\begin{array}{ccc}v & 0 & 0 \\ 0 & v & -\omega \\ 0 & \omega & v\end{array}\right)$ $V I-15$

avec $\omega=\frac{q B}{m}$ la gyrofréquence (voir équation $I I I-16$ ), soit la représentation suivante de $\underline{\underline{\mu}}$ : 
$\underline{\underline{\mu}}=\frac{1}{m}\left(\begin{array}{ccc}\frac{1}{v} & 0 & 0 \\ 0 & \frac{v}{v^{2}+\omega^{2}} & \frac{\omega}{v^{2}+\omega^{2}} \\ 0 & -\frac{\omega}{v^{2}+\omega^{2}} & \frac{v}{v^{2}+\omega^{2}}\end{array}\right)=\frac{1}{q B}\left(\begin{array}{ccc}\frac{\omega}{v} & 0 & 0 \\ 0 & \frac{\omega v}{v^{2}+\omega^{2}} & \frac{\omega^{2}}{v^{2}+\omega^{2}} \\ 0 & -\frac{\omega^{2}}{v^{2}+\omega^{2}} & \frac{\omega v}{v^{2}+\omega^{2}}\end{array}\right)$

L'introduction du tenseur nous permet de récrire l'équation $V I-14$ sous la forme :

$\underline{\underline{\mu}}^{-1} \vec{u}=\vec{F}$

et donc d'exprimer simplement la solution de l'équation du mouvement :

$\vec{u}=\underline{\underline{\mu}} \vec{F}$

$V I-18$

$\underline{\underline{\mu}}$ est appelé le tenseur de mobilité $\left[\mathrm{s} . \mathrm{kg}^{-1}\right]$, et il relie de manière linéaire la cause (la force $\vec{F}$ ) à l'effet (le déplacement à la vitesse $\vec{u}$ ). Cette écriture formelle est indépendante de la base orthonormée directe. Seules les équations projetées changent et dans ce cas, une opération de changement de base appliquée à la matrice représentant le tenseur $\underline{\underline{\mu}}$ permet d'obtenir la représentation de $\underline{\underline{\mu}}$ dans une autre base orthonormée directe et par là, d'obtenir les composantes de la solution. Dans la limite $\omega<<v$, le tenseur $\underline{\underline{\mu}}$ se réduit à la mobilité scalaire $\mu=\frac{1}{v m}$.

Si nous nous intéressons aux mouvements en région $E$ des ions et des électrons soumis à l'action du champ électrique (nous prendrons $\vec{F}=q \vec{E}$ ), où $\vec{E}$ est le champ électrique d'origine magnétosphérique qui se projette dans l'ionosphère (voir chapitre $I I I$ et paragraphe suivant), nous pouvons écrire :

$$
\left\{\begin{array}{l}
\overrightarrow{u_{e}}=-\underline{\underline{\mu}}_{e} e \vec{E} \\
{\overrightarrow{u_{i}}}^{=} \underline{\underline{\mu}}_{i} e \vec{E}
\end{array}\right.
$$

où nous avons caractérisé le fait que les ions et les électrons se déplacent avec leur propre mobilité. $e$ est la charge élémentaire. De fait, les ions et les électrons ont des vitesses différentes et une densité de courant $\left(\mathrm{A} . \mathrm{m}^{-2}\right)$ apparaît donc dans l'ionosphère qui vaut :

$\vec{j}=n_{e} e\left(\overrightarrow{u_{i}}-\overrightarrow{u_{e}}\right)$

soit, en utilisant VI-19:

$\vec{j}=n_{e} e^{2}\left(\underline{\underline{\mu_{i}}}+\underline{\underline{\mu}}_{e}\right) \vec{E}$ VI-21 
Ce courant boucle par le bas le système des courants de Birkeland que nous avons examiné dans le chapitre III. Il constitue un couplage direct entre la magnétogaine aurorale et l'ionosphère. Il est donc un témoin très sensible de l'activité électrique dans la magnétosphère, et par conséquent de l'activité solaire. Il constitue l'électrojet auroral, déjà présenté au chapitre III. On introduit $\underline{\underline{\sigma}}$ le tenseur de conductivité :

$\underline{\underline{\sigma}}=n_{e} e^{2}\left(\underline{\underline{\mu_{i}}}+\underline{\underline{\mu_{e}}}\right)$

et on peut alors écrire une loi d'Ohm :

$\vec{j}=\underline{\underline{\sigma}} \vec{E}$

$V I-23$

Le courant dans la direction de $\vec{E}$ s'appelle le courant de Pedersen, tandis que celui dans la direction perpendiculaire à la fois à $\vec{E}$ et à $\vec{B}$ s'appelle le courant de Hall. Les conductivités correspondantes ont pour expression :

$$
\left\{\begin{array}{l}
\sigma_{P}=\frac{n_{e} e}{B}\left(\frac{\omega_{i} v_{i n}}{v_{i n}^{2}+\omega_{i}^{2}}+\frac{\left|\omega_{e}\right| v_{e n}}{v_{e n}^{2}+\omega_{e}^{2}}\right) \\
\sigma_{H}=\frac{n_{e} e}{B}\left(\frac{\omega_{e}^{2}}{{v_{e n}}^{2}+\omega_{e}^{2}}-\frac{\omega_{i}^{2}}{v_{i n}^{2}+\omega_{i}^{2}}\right)
\end{array}\right.
$$

Enfin, la conductivité parallèle ou longitudinale s'exprime de la manière suivante :

$$
\sigma_{/ /}=\frac{n_{e} e}{B}\left(\frac{\omega_{i}}{v_{i n}}+\frac{\left|\omega_{e}\right|}{v_{e n}}\right)
$$

On les exprime en mho. $\mathrm{m}^{-1}$. mho est le mot Ohm à l'envers, pour rappeler que les conductivités sont l'inverse de résistances. Dans ces expressions, nous n'avons considéré que les collisions des ions et des électrons avec les espèces neutres, qui sont prépondérantes devant les collisions entre espèces chargées en région $E$. D'autre part, d'après la définition que nous avons donné de la gyrofréquence, celle-ci est négative pour les électrons, d'où la présence de la valeur absolue. Plus l'altitude est élevée et moins il y a de collisions, les densités devenant de plus en plus faibles. L'expression $V I-25$ laisserait alors penser que la conductivité parallèle tend vers l'infini. Ceci est bien sûr inexact : à haute altitude, les collisions coulombiennes électrons-ions deviennent prépondérantes. Le courant dépend alors du rapport $\frac{n_{e}}{v_{e i}}$. Comme $v_{e i}$ est proportionnelle à la densité électronique, le courant cesse de dépendre de $n_{e}$. La figure VI-7 montre les conductivités Hall et Pedersen à haute latitude dans les mêmes conditions que dans le chapitre $V$ : une latitude moyenne (celle d'une ville comme Grenoble, c'est à dire à 45 degrés de latitude nord), un jour de Juillet 1998 (jour 205), à midi dans des conditions magnétiques calmes.

La conductivité Pedersen est supérieure à la conductivité Hall au dessus d'environ $120 \mathrm{~km}$. En fait, seule la région 100-140 km d'altitude présente une conductivité importante, perpendiculairement au champ magnétique. On l'appelle alors parfois la couche de conductivité 
perpendiculaire élevée. En dessous de cette région, la concentration électronique est très faible, et il en est donc de même de la conductivité. Au-dessus de cette région, $v_{i n}$ et $v_{e n}$ sont beaucoup plus petites que $\omega_{i}$ et $\omega_{e}$ et la conductivité est faible car électrons et ions se déplacent simultanément. C'est donc dans la région $E$, de conductivité importante, que s'écoulent les courants horizontaux liés aux mouvements de l'ionisation car la gyrofréquence y est plus faible (ou du même ordre de grandeur) que les fréquences de collisions, pour toutes les espèces et des mouvements différents selon la charge peuvent exister perpendiculairement aux lignes de champ. Par contre au-dessus de cette région, comme la gyrofréquence devient plus importante que les fréquences de collisions pour toutes les espèces chargées, les mouvements de particules se font le long des lignes de champ magnétique autour desquelles ces particules sont piégées; les courants sont donc les courants alignés de Birkeland, qui se referment alors en région $E$ par ces courants horizontaux.

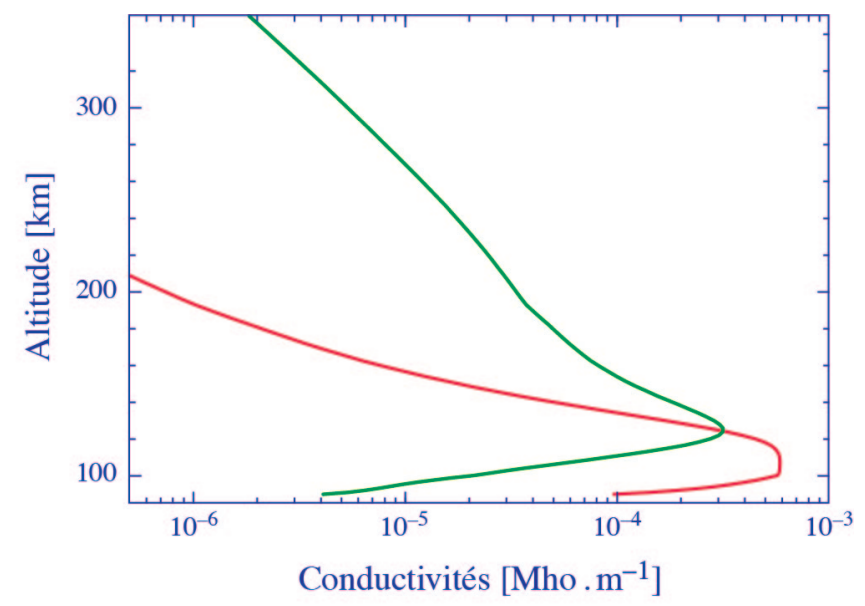

Figure VI-7 : conductivités Hall (rouge) et Pedersen (vert) en fonction de l'altitude

Les valeurs intégrées en altitude s'appellent les conductivités intégrées, ou conductances, et sont notées $\Sigma$. Ainsi dans le cas présenté ci-dessus, la conductance Hall vaut 19,1 mho, et la conductance Pedersen 14,1 mho. Le rapport est donc de 1,35. On donne toujours le rapport des conductances en terme Hall/Pedersen; il indique l'état d'anisotropie électrique de l'ionosphère, puisque la conductivité Pedersen est dans la direction du champ électrique $\vec{E}$ (perpendiculaire au champ magnétique $\vec{B}$ ), et la conductivité Hall est perpendiculaire à la fois à $\vec{E}$ et à $\vec{B}$.

Remarquons que la mesure de $n_{e}$ suffit à la connaissance des conductivités. Cette mesure est possible avec des radars ionosphériques, et la déduction de $\sigma$ est immédiate (les fréquences de collisions sont données par les formules du chapitre $V$ ). Une mesure supplémentaire du champ électrique permet d'accéder aux courants ionosphériques, et donc à la différence des vitesses ioniques et électroniques. On comprend que les conductivités Hall et Pedersen sont des paramètres indispensables à la compréhension des phénomènes 
ionosphériques et du couplage ionosphère/ magnétosphère. La figure VI-8 montre les conductances basées sur les mesures de densités électroniques pendant trois années (1989 à 1991). Elles correspondent à des conditions solaires calmes : $\mathrm{f}_{10.7}$ moyen de $80, \mathrm{Kp}$ moyen de 3 , correspondant à $\mathrm{Ap}=14$. Les conductivités ionosphériques Pedersen et Hall sont calculées suivant les formules $V I-24$.

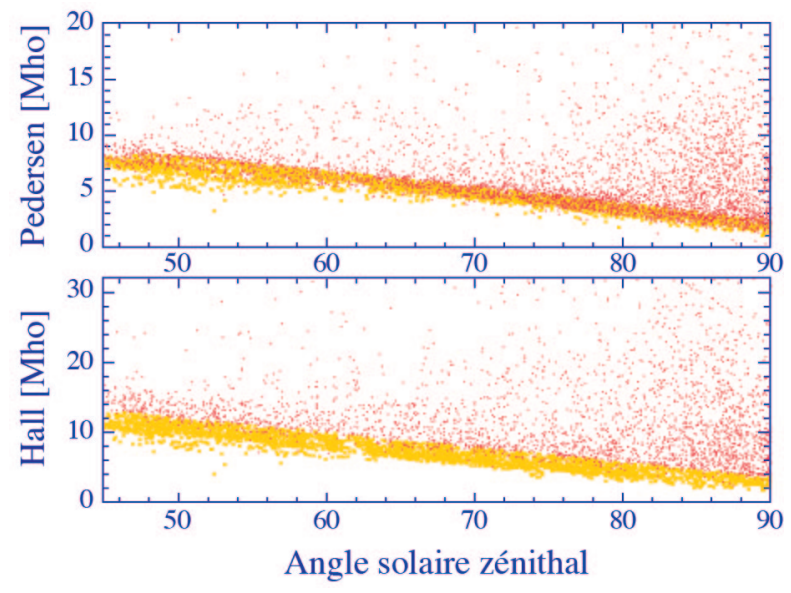

Figure $V I-8$ : conductances Hall et Pedersen déduites de trois années de mesures radar

Les croix jaunes sont attribuées à des conductivités diurnes, électrons issus de la photoionisation, tandis que les points rouges sont attribués aux conductivités nocturnes, électrons dus à l'entrée du vent solaire. (Senior, C., Solar and particle contributions to auroral height-integrated conductivities from EISCAT data : a statistical study, Ann. geophysica, 9, 449-460, 1991).

On constate que les conductances Hall sont plus linéaires (en fonction de l'angle solaire zénithal) que les conductances Pedersen, avec des valeurs plus élevées d'environ 2 mho.

La nuit, les conductances sont intimement liées aux précipitations, sources de l'ionosphère. L'altitude de la production électronique dépend de l'énergie caractéristique des précipitations; les conductances nocturnes sont donc plus ou moins élevées selon que cette énergie est plus ou moins grande. Or, la couche de conductivité se trouve en dessous de $120 \mathrm{~km}$; la figure VI-4 montre que les précipitations qui augmentent les conductances doivent donc être d'énergie supérieure à $2 \mathrm{keV}$. Il s'agit de ce que nous avons défini comme la première population des électrons précipités, avec un maximum nocturne après minuit. Cela se reconnaît sur la figure VI-9. Il s'agit d'une moyenne établie sur 11 ans de données nocturnes (1300 heures d'observation).

L'amplitude des conductances nocturnes est tout à fait comparable à celle des amplitudes diurnes, avec un rapport Hall / Pedersen de l'ordre de 1,4. Comme les conductances Hall et Pedersen n'ont pas leur maximum à la même altitude, leur rapport nocturne est un indicateur de l'énergie des précipitations. Ainsi, un rapport voisin de 1 indique-t-il des précipitations d'énergie de l'ordre de $2,5 \mathrm{keV}$, tandis qu'un rapport proche de 2 témoigne de précipitations de l'ordre de $6 \mathrm{keV}$. Cependant, cet indicateur ne donne qu'une valeur 
extrêmement approchée, dans la mesure où il ne tient pas compte de la nature des particules précipitantes : électrons ou protons.
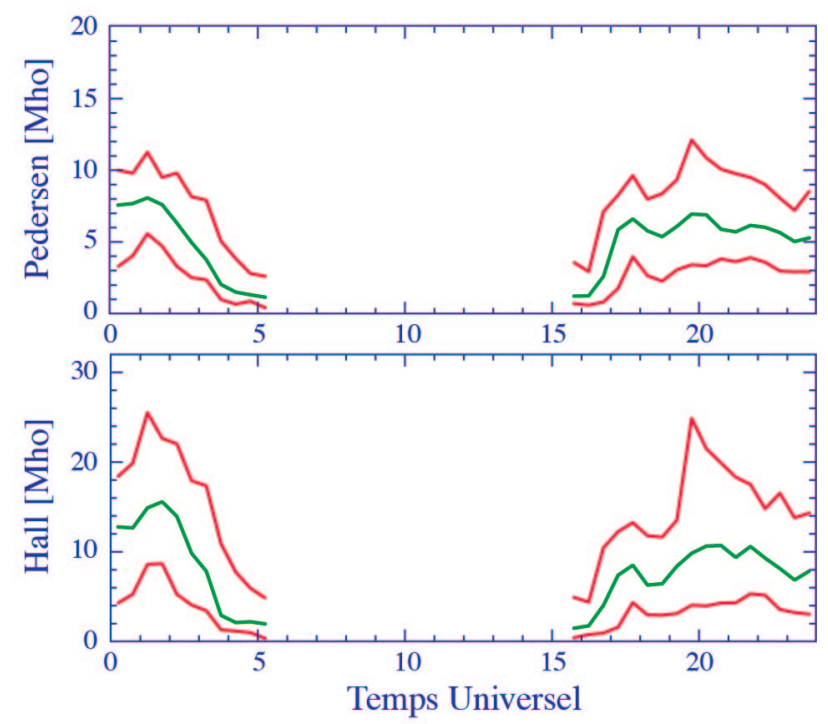

Figure VI-9 : conductances nocturnes Hall et Pedersen déduites de onze années de mesures radar. En vert, les moyennes, en rouge, les valeurs inférieures et supérieures.

(J.A. Davis et M. Lester, Université de Leicester, communication personnelle).

La connaissance des conductivités ionosphériques va nous permettre de décrire l'electrojet équatorial qui est un courant ionosphérique observé en région équatoriale. Le phénomène résulte du fait qu'à l'équateur, le champ magnétique est horizontal, orienté du nord vers le sud. En choisissant un repère orthonormé direct dont la première direction est parallèle au champ magnétique (composante repérée par l'indice //, comme précédemment), la seconde est orientée vers l'est (composante $y$, tangente à l'équateur magnétique) et la troisième est confondue avec la verticale (composante z), la projection de la loi d'Ohm VI-23 nous donne :

$$
\left\{\begin{array}{l}
j_{/ /}=\sigma_{/ /} E_{/ /} \\
j_{y}=\sigma_{P} E_{y}-\sigma_{H} E_{z} \\
j_{z}=\sigma_{P} E_{z}+\sigma_{H} E_{y}
\end{array}\right.
$$

Le champ électrique équatorial est généralement orienté est-ouest, et l'ionosphère peut être considérée comme stratifiée horizontalement. Les courants verticaux sont suffisamment faibles pour pouvoir être négligés $\left(j_{z}=0\right)$. Nous obtenons le courant dans la direction estouest :

$j_{y}=\left(\sigma_{P}+\frac{\sigma_{H}^{2}}{\sigma_{P}}\right) E_{y}$ 
que l'on on écrit conventionnellement sous la forme suivante :

$j_{y}=\sigma_{c} E_{y}$

où $\sigma_{c}$ est la conductivité de Cowling. A l'équateur, la conductivité Hall possède un pic large autour de $130 \mathrm{~km}$, avec une valeur typique de l'ordre de $410^{-4}$ mho.m ${ }^{-1}$. Vers $150 \mathrm{~km}$ comme vers $90 \mathrm{~km}$, elle tend vers zéro. La conductivité Pedersen possède pour sa part un pic étroit vers $150 \mathrm{~km}$ d'amplitude typique de $310^{-4}$ mho.m ${ }^{-1}$. Vers $220 \mathrm{~km}$ comme vers $100 \mathrm{~km}$, elle est quasiment nulle. La conductivité de Cowling qui en résulte possède un pic étroit vers $100 \mathrm{~km}$, d'amplitude de l'ordre de $810^{-3}$ mho.m ${ }^{-1}$. En dessous du pic, elle tend vers zéro en une quinzaine de kilomètres. Au-dessus, elle décroît lentement jusqu'à $160 \mathrm{~km}$. Horizontalement, le champ électrique équatorial est de l'ordre de $0,5 \mathrm{mV} \cdot \mathrm{m}^{-1}$. Il résulte de ces valeurs un courant équatorial de région $E$ appelé l'électrojet équatorial. Il s'écoule le long de l'équateur magnétique à environ $100 \mathrm{~km}$, sur une bande de quelques degrés de latitude. Le jour, il est orienté vers l'est, et il va vers l'ouest la nuit. Cependant, la faible concentration électronique nocturne le rend difficilement mesurable. Le système des électrojets est montré sur la figure $V I-10$.

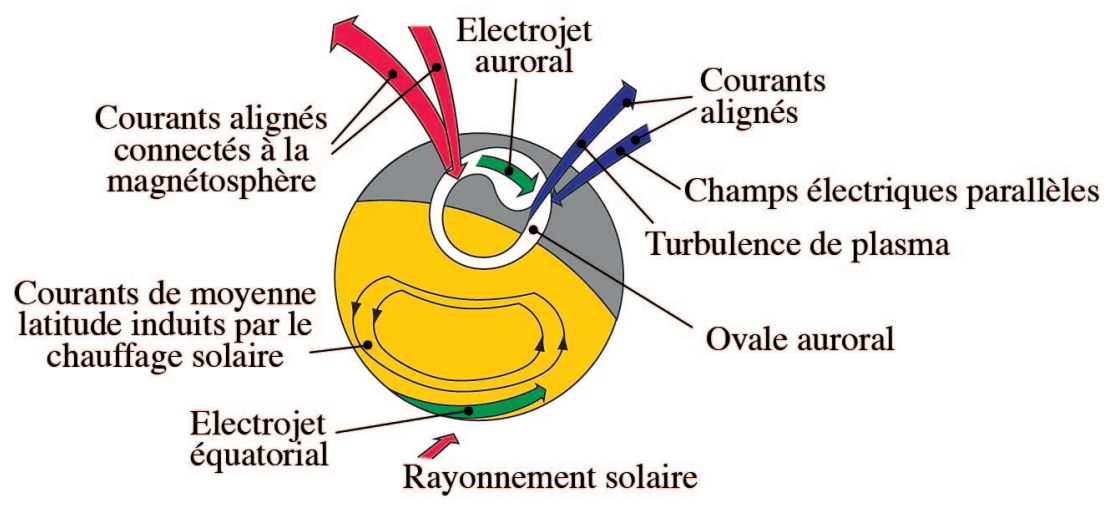

\section{Figure $V I-10$ : configuration des principaux systèmes de courants de l'ionosphère terrestre}

Dans cette discussion, nous avons souligné que le champ électrique équatorial horizontal est-ouest est important, et que le champ magnétique est lui-même horizontal (nord-sud). Il en résulte une vitesse de dérive des ions et électrons perpendiculaires aux deux champs, c'est à dire dans le sens vertical. Le principe de cette dérive a été étudié au chapitre III sur la magnétosphère terrestre. La convection qui en résulte dans la région $F$ est vers le haut et vers l'ouest le jour, et vers le bas et vers l'est la nuit. Le jour, du plasma est transporté de $200 \mathrm{~km}$ jusqu'à environ $1000 \mathrm{~km}$, altitude à laquelle la recombinaison est lente. Ce plasma est transporté vers les latitudes plus élevées (jusqu'environ $25^{\circ}$ ), où il se retrouve en basse région $E$ sous l'effet de la gravité. La figure $V I-11$ montre la mesure de ces flux, qui ont reçu le joli nom de fontaine équatoriale. Nous n'entrerons pas plus avant dans son étude. Soulignons simplement que ce flux de plasma interagit avec le vent neutre, si bien qu'il n'est pas parfaitement symétrique autour de l'équateur magnétique, et qu'enfin le décalage 
entre l'équateur magnétique et l'équateur géographique (et donc l'éclairement solaire) crée des variations saisonnières de cette fontaine équatoriale.

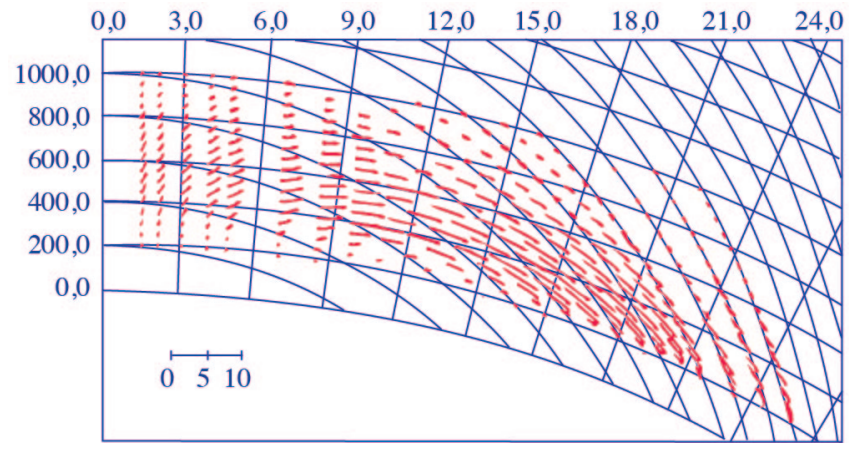

Figure VI-11 : mesure des flux de la fontaine équatoriale (Townsend, 1982)

La latitude magnétique est portée sur l'abscisse supérieure, de 0 à 24 degrés.. L'altitude (de 0 à $1000 \mathrm{~km}$ ) est sur l'ordonnée à gauche. La barre d'échelle au bas de la figure correspond à $10^{9} \mathrm{~cm}^{-2} \cdot \mathrm{s}^{-1}$.

\section{LE CHAMP ÉLECTRIQUE DES HAUTES LATITUDES}

Les dernières théories développées pour expliquer l'interaction entre le vent solaire et le champ magnétique terrestre font appel à la notion de reconnexion. Bien qu'elle n'ait pas formellement été mise en évidence, la reconnexion semble en effet la cause la plus simple pour expliquer les mouvements du plasma observés aux hautes latitudes et que nous allons présenter dans ce paragraphe.

\section{a. Principe de la reconnexion}

Un modèle MHD idéal d'interaction entre le vent solaire et le champ magnétique interdit tout échange de matière entre les deux systèmes, ainsi que nous l'avons vu au chapitre $I I I$. La séparation entre ces deux systèmes se fait par l'intermédiaire d'une nappe de courant qui drape la magnétopause, la surface séparatrice entre les champs magnétiques interplanétaire et terrestre. Le courant est très intense et peut s'avérer être instable dans les conditions de gradient de champ magnétique observées à la magnétopause. Lorsque les champs magnétiques ont des directions opposées, ce qui signifie que le champ magnétique interplanétaire est orienté Nord-Sud, l'un des modes possible d'instabilité est le mode de déchirement qui aboutit à une rupture de la surface séparatrice et à une reconnexion des champs magnétiques de part et d'autre de la surface ; c'est le principe de la magnétosphère de Dungey que nous avons mentionné au chapitre III. 


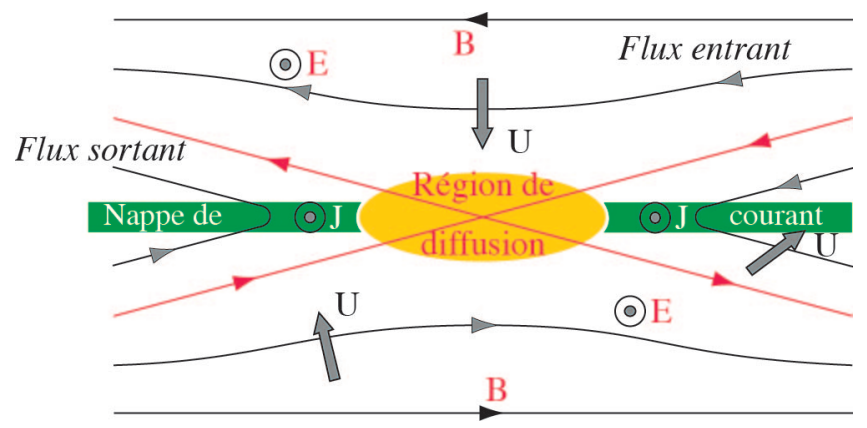

Figure VI-12 : illustration de la reconnexion magnétique en un point neutre de type $X$

La zone verte est la nappe de courant séparant les champs magnétiques de sens inverse. La zone jaune est la région de diffusion à travers laquelle les particules peuvent passer d'un système à l'autre ; c'est la zone de reconnexion.

Le mode le plus simple est la reconnexion de type $\mathrm{X}$ (nom donné à cause de la configuration des lignes de champs au point de reconnexion) qui consiste à couper deux lignes de champ (une dans chaque région) en un point et à les reconnecter au travers de la surface séparatrice (figure $V I-12$ ). Le point où s'opère cette reconnexion est dit point neutre de type $\mathrm{X}$. A la suite de cette reconnexion, la magnétosphère n'est plus isolée et des particules du vent solaire peuvent pénétrer dans le système magnétosphérique par la région de diffusion. On arrive ainsi à la configuration présentée sur la figure $V I-13$.

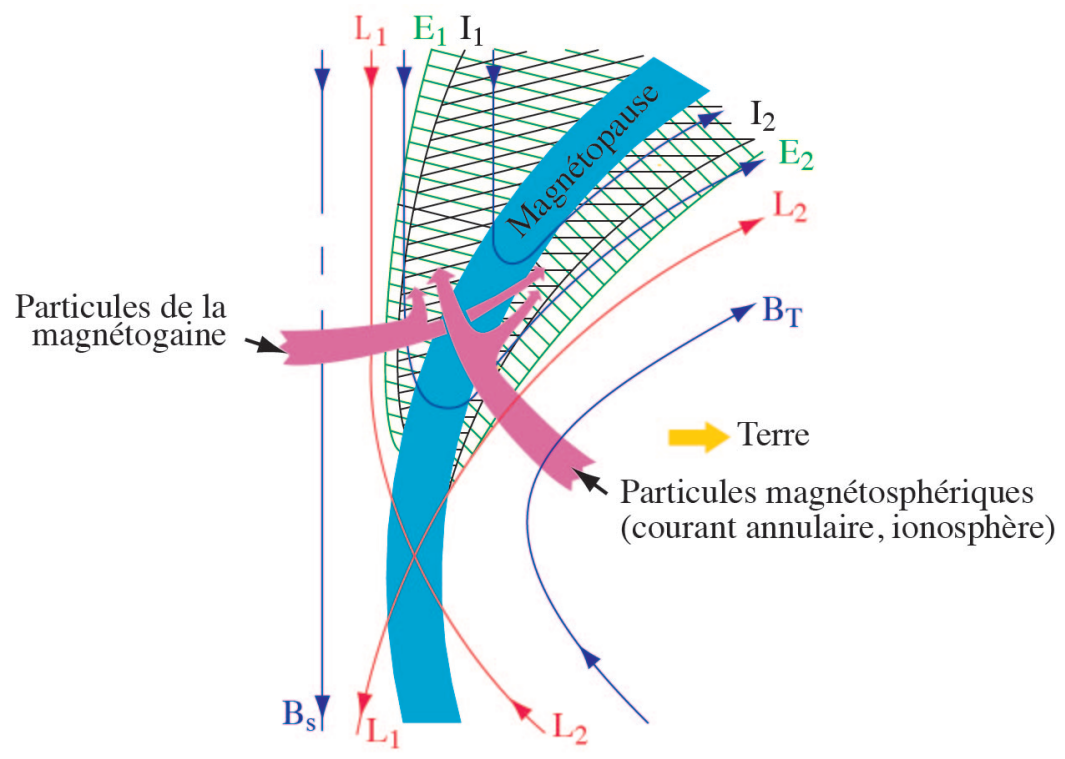

Figure $V I-13$ : illustration décrivant la géométrie de la reconnexion sur la face avant de la magnétosphère en un point neutre $X$. Les transferts de particules depuis la magnétogaine et la magnétosphère sont indiqués par les grosses flèches. Les lignes de champ marquées $S_{l}$ et $S_{2}$ sont les lignes concernées par la reconnexion. 
Les lignes de champ interplanétaire se déplacent dans l'espace suite à l'expansion que nous avons décrite au chapitre III. De fait, ce point de reconnexion se déplace sur la surface de la magnétopause et décrit alors une ligne que l'on appelle la ligne neutre.
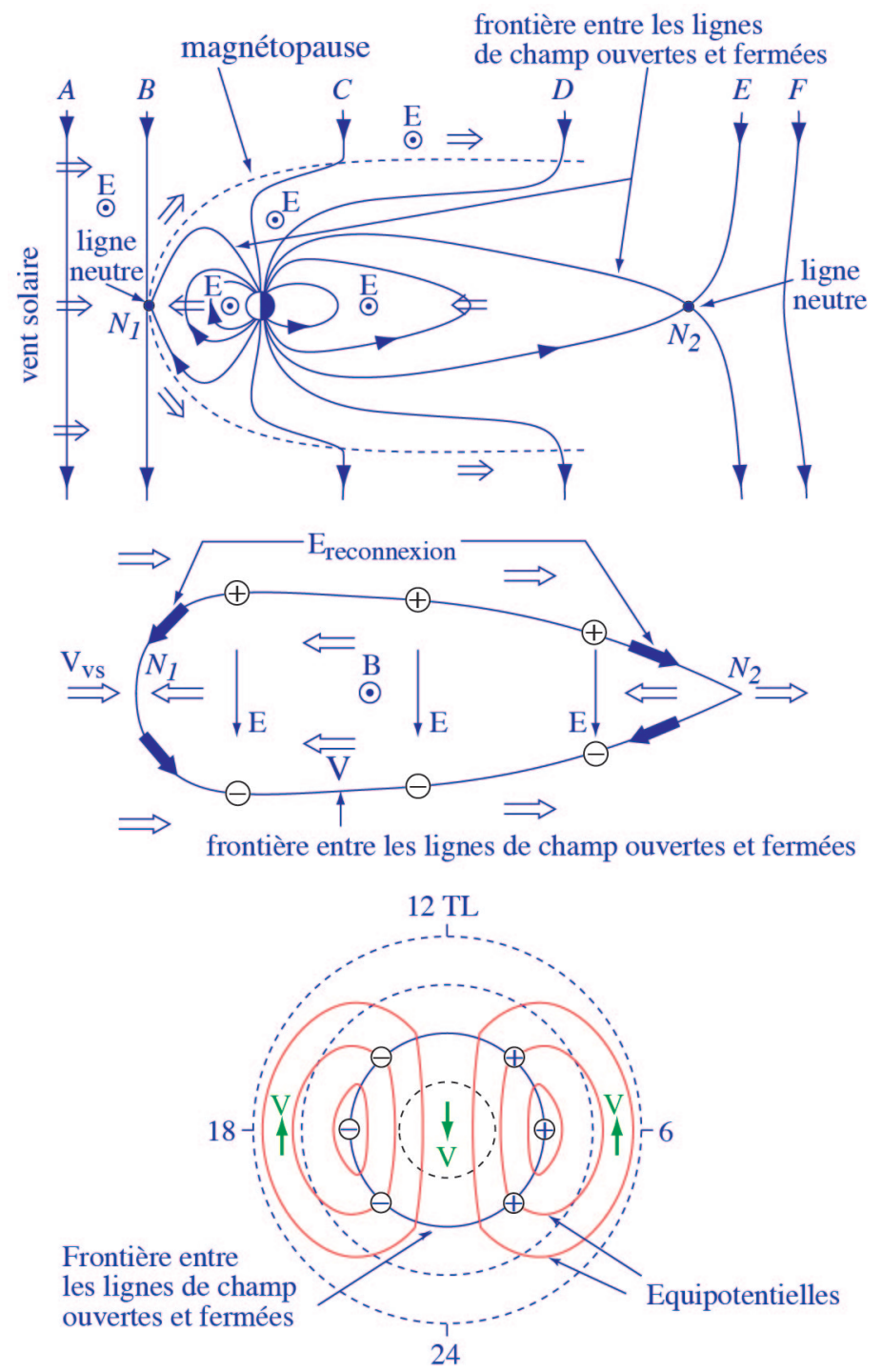

Figure VI-14 : principe du transport des lignes de champs

et du plasma dans le modèle de magnétosphère ouverte

L'interconnexion des lignes de champs (a) définit une ligne neutre (b) à l'intérieur de laquelle le plasma est entraîné du côté jour vers le côté nuit ; à l'extérieur de cette ligne, c'est le phénomène inverse qui se produit. La projection en région aurorale de cette ligne neutre correspond à l'ovale auroral (c) qui délimite la direction de la convection du plasma ionosphérique organisée en deux vortex contrarotatoires : à l'intérieur de l'ovale convection dans la direction de minuit, convection dans la direction inverse à l'extérieur. 
La figure VI-14 représente ce transport des lignes de champ interplanétaire (succession $A \rightarrow F$ sur la figure (a) qui sont supposées se reconnecter avec le champ magnétique terrestre au point $\mathrm{N}_{l}$ sur la face avant de la magnétosphère et décrire la ligne neutre (figure (b) entre le point $\mathrm{N}_{1}$ et le point $\mathrm{N}_{2}$. La symétrie de la magnétosphère par rapport au plan du dipôle magnétique terrestre dans la direction midi-minuit entraîne la présence de deux lignes symétriques par rapport à ce plan. Au-delà du point $\mathrm{N}_{2}$ la reconnexion ne peut plus être assurée et les lignes de champ se déconnectent : les lignes de champ interplanétaire continuent leur mouvement d'expansion et les lignes de champ terrestre se referment dans la magnétosphère.

Le mécanisme de reconnexion définit alors trois classes topologiques de lignes de champ magnétiques :

- la classe 1 correspond aux lignes de champ interne à la magnétosphère qui sont reliées à la Terre en leurs deux extrémités et ont un mouvement associé à la corotation de la Terre ;

- la classe 2 correspond aux lignes de champ reconnectées; ces lignes ont donc une extrémité reliée à la Terre et une extrémité plongée dans le milieu interplanétaire qui s'écoule depuis le côté jour vers le côté nuit ;

- la classe 3 correspond aux lignes de champ interplanétaire entièrement plongées dans le vent solaire.

Les lignes de champ magnétosphérique qui reconnectées rencontrent l'équateur magnétique loin de la Terre sur la face avant. Elles correspondent alors à un paramètre de Mac-Illwain $L$ (équation $I I-76$ ) élevé et ont donc leurs pieds immergés dans l'ionosphère aux hautes latitudes, dans les régions polaires. D'après les mesures satellitaires, il semble à peu près clair qu'au niveau de l'ionosphère, la région de séparation entre les lignes des classes 1 et 2 correspond à l'ovale auroral. Les deux ovales auroraux apparaissent ainsi comme les projections (dans chaque hémisphère) dans l'ionosphère, le long des lignes de champ magnétique, de la ligne neutre introduite précédemment.

\section{b. Convection des lignes de champ et du plasma magnétosphérique}

Les lignes de la classe 2 ont un mouvement de convection dans la direction jour-nuit induit par l'expansion du vent solaire. Les conditions de la MHD idéale (entre autre hypothèse du flux gelé) ne sont remises en cause que dans la région de reconnexion (donc le long de la ligne neutre) et restent valables partout ailleurs dans la magnétosphère. Par conséquent, l'hypothèse majeure du flux gelé implique que les lignes de champ entraînent le plasma magnétosphérique dans leur mouvement de convection. Le long des lignes de champ reconnectées, le plasma s'écoule donc du côté jour vers le côté nuit, ce qui est traduit par les flèches dans la direction antisolaire sur la figure VI-14. Cependant, nous avons conservation du flux magnétique et donc, pour que le mécanisme de reconnexion puisse être permanent sur la face avant de la magnétosphère, il doit exister un mouvement de convection dans la direction nuit-jour qui contrebalance la convection antisolaire ; cette convection ne peut toutefois avoir lieu que le long des lignes de champ de la classe 1. En fait, dans le plan de l'équateur, on a montré expérimentalement que le plasma a un 
mouvement de convection depuis le côté nuit vers le côté jour. La séparation entre les deux types de convection est alors la ligne neutre.

$\mathrm{Si}$ on projette cette convection globale le long des lignes de champ depuis le plan équatorial magnétique vers l'ionosphère des hautes latitudes, on trouve que le plasma ionosphérique doit avoir un mouvement de convection compatible avec le schéma de la figure $V I-14 c$. Le mouvement se fait dans la direction antisolaire à l'intérieur de l'ovale auroral (qui est la projection de la ligne neutre) tandis qu'il est dans la direction solaire à l'extérieur de cet ovale. La symétrie magnétique entraîne alors l'apparition de deux vortex contrarotatoires dans chaque hémisphère, le long desquels s'organise le mouvement du plasma : on l'appelle convection magnétosphérique.

Toutefois, cette description n'est valable que dans l'hypothèse où la Terre est fixe. Si on rajoute la rotation de la planète, on complique le mouvement du plasma à l'intérieur de la magnétosphère. En effet, le champ électrique magnétosphérique qui contraint la convection du plasma à l'intérieur de la magnétosphère se trouve alors en compétition avec le champ de corotation de la Terre et le mouvement réel du plasma est la superposition des deux champs (voir figure $I I I-9$ pour la trace de la combinaison des deux champs dans le plan équatorial magnétique). On aboutit donc à un schéma de convection global qui définit deux régions distinctes :

- une région où la rotation de la Terre contraint le mouvement du plasma ;

- une région où la convection magnétosphérique contraint le mouvement du plasma.

La première région définit la plasmasphère, que nous avons introduite au chapitre $I I I$, qui apparaît alors comme une région de tubes de champ fermés où le plasma est en équilibre de pression, et qui se déplace avec la Terre.

La deuxième région correspond à ces tubes de champs qui sont connectés à l'environnement interplanétaire et pour lesquels nous pouvons considérer qu'ils sont ouverts sur le vide. L'équilibre de pression n'y est jamais réalisé et on peut assister à un échappement de particules le long de ces lignes de champ ; c'est le mécanisme du vent polaire que nous décrirons plus loin. On caractérise souvent cette région comme une région de trou de concentration pour les ions légers.

Entre ces deux régions existe une classe de lignes de champ fermées qui sont plus ou moins entraînées par la convection magnétosphérique. Dans l'équilibre qui s'instaure entre le champ magnétique interplanétaire et le champ magnétique terrestre, ces lignes de champ ont une projection dans l'ionosphère qui se situe à haute latitude, près de la région aurorale et une projection dans le plan de l'équateur magnétique qui se situe loin dans la queue. Plus on monte en latitude, plus la convection l'emporte sur la corotation et plus la projection dans le plan équatorial s'éloigne.

Il existe une région où la corotation compense exactement la convection ; le plasma qui s'y trouve est maintenu indéfiniment dans cette région (dans une hypothèse de stabilité de la convection). Comme cette région se trouve dans le côté soir (voir figure III-9), les mécanismes chimiques de recombinaison sont prépondérants sur les mécanismes de création par photoionisation et cette région est donc caractérisée par un trou de densité de plasma. 


\section{c. Champ électrique de convection}

Depuis le début de ce paragraphe, nous associons la trace dans l'ionosphère à celle dans le plan équatorial magnétique, car elles sont les deux faces d'un même phénomène. Dans le chapitre $I I I$, nous avons introduit le champ électrique qui naît de l'interaction entre le champ magnétique terrestre et le vent solaire. Ce champ électrique est responsable de la convection du plasma dans la magnétosphère et doit avoir son pendant dans l'ionosphère pour expliquer la convection du plasma ionosphérique.

En effet, la convection du plasma que l'on observe dans l'ionosphère en régions aurorale et polaire n'est possible que si une force impose un mouvement au plasma. Comme l'atmosphère neutre est insensible (initialement) à cette convection, la force doit être d'origine électrique. Il apparaît alors que le champ électrique qui impose la convection ionosphérique du plasma est la projection le long des lignes de champ, que l'on suppose conductrices, du champ électrique présent dans le plan équatorial magnétique. Ce champ électrique de convection dans l'ionosphère est donc une caractérisation de fait du couplage qui existe entre la magnétosphère et le vent solaire.

Pour des raisons de simplicité, nous supposerons que l'atmosphère neutre est au repos et qu'elle n'a donc aucun mouvement. Les particules (de charge $q$ ) du plasma ionosphérique qui sont baignées dans le champ magnétique terrestre $\vec{B}$ sont soumises à la force de Lorentz dont l'expression dans le référentiel terrestre est :

$\vec{F}=q\left(\overrightarrow{E_{\perp}}+\vec{v} \wedge \vec{B}\right)$

où $\vec{v}$ est la vitesse de convection et $\overrightarrow{E_{\perp}}$ le champ de convection ionosphérique. L'hypothèse de flux gelé de la MHD idéale impose que le champ électrique dans le référentiel lié au mouvement des ions est nul ou, ce qui revient au même, que la force de Lorentz dans le référentiel terrestre est nulle. On en déduit alors la relation entre la convection et le champ électrique :

$\overrightarrow{E_{\perp}}=-\vec{v} \wedge \vec{B}$

$V I-30$

La solution :

$\vec{v}=\frac{\overrightarrow{E_{\perp}} \wedge \vec{B}}{B^{2}}$

$V I-31$

correspond à la vitesse de dérive dont nous avons déjà discuté au chapitre $I I I$ (équation III-24), avec cette différence que nous nous trouvons dans l'ionosphère et non dans la magnétosphère. Ainsi que nous l'avions déjà remarqué, cette vitesse est la même pour toutes les particules, puisqu'elle ne dépend que des champs extérieurs et pas de la charge.

Cette convection se fait dans une direction qui est à la fois perpendiculaire à $\overrightarrow{E_{\perp}}$ et à $\vec{B}$ et on l'appelle donc dérive $E$ croix $B$. On en déduit donc que le mouvement des particules est organisé le long des équipotentielles du potentiel électrostatique dont dérive le champ $\overrightarrow{E_{\perp}}$ et dans un plan perpendiculaire au champ magnétique. 
Dès lors, on peut aisément déterminer la structure du potentiel électrostatique dans l'ionosphère à partir de la mesure des vitesses des ions dans une région où les conditions de la MHD sont vérifiées, condition qui est remplie au-dessus de $120 \mathrm{~km}$ typiquement.

Le radar EISCAT-UHF tristatique permet d'accéder à la convection du plasma en région aurorale entre 62 et $72^{\circ}$ de latitude invariante. A partir de la base de données de ce radar, un modèle statistique de convection a pu être développé. La figure VI-15 représente la cartographie des lignes équipotentielles du champ électrique de convection pour une période agitée, déterminée à partir de ce modèle.

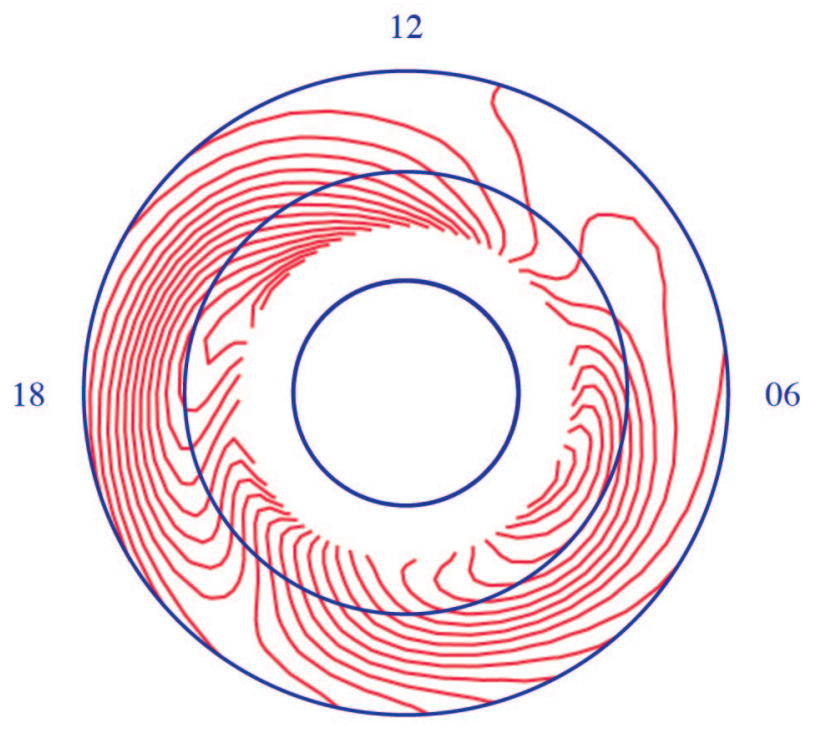

00

Figure VI-15 : carte des équipotentielles (en rouge) du champ électrique de convection en région aurorale entre $60^{\circ}$ et $72^{\circ}$ de latitude invariante pour une période active $(\mathbf{K p}>\mathbf{6})$. Le tracé est effectué en coordonnées magnétiques avec le midi local (12) dirigé vers le soleil et le minuit local dirigé dans la direction antisolaire. On a représenté les cercles de latitudes $60^{\circ}, 70^{\circ}$ et $80^{\circ}$ (en bleu).

\section{LE COUPLAGE ÉNERGÉTIQUE}

La réalité du couplage électrodynamique entre l'ionosphère et la magnétosphère est manifeste sur le bilan énergétique des populations du plasma ionosphérique. En effet, malgré une conductivité élevée, l'ionosphère est néammoins un milieu résistif qui dissipe l'énergie électrique sous forme de chauffage Joule ; la manière dont cette énergie est dissipée varie selon qu'il s'agit d'ions ou d'électrons. Nous allons décrire trois mécanismes différents qui contribuent au chauffage de l'ionosphère et qui montrent la diversité de ce couplage. 


\section{a. L'action du champ électrique}

\section{i. Chauffage ionique par friction}

Lorsque nous avons introduit l'équation du bilan d'énergie à l'équation $V-143$, nous avons présenté le terme d'échange thermique dans lequel nous avons fait figurer deux contributions. La première a été discutée dans le cas de l'ionosphère diurne est reste évidemment valable pour l'ionosphère des régions aurorales. La seconde contribution mérite d'être détaillée. En effet, la théorie qui conduit à l'établissement des équations de transport utilise la méthode des perturbations pour établir les termes de collisions, qui sont donc des développements à l'ordre 1 . Ainsi, dans le cas de l'équation d'évolution de l'énergie, nous ne devrions avoir que la première contribution qui correspond à un réel échange thermique. Cependant, si nous reprenons la description du chapitre $I V$, nous pouvons affirmer qu'une collision a pour effet principal de décollimater un faisceau, car elle introduit une déflexion des particules. Or que représente l'énergie thermique, si ce n'est la dispersion en énergie du faisceau? Un faisceau monoénergétique aurait une température nulle, mais s'il subit une déflexion au cours de collisions, la décollimation entraîne une dispersion en vitesse et en énergie de telle sorte qu'après l'événement collisionnel, la température du faisceau est non nulle. Les collisions ont donc fait gagner de l'énergie thermique au faisceau au détriment de son énergie cinétique ; le faisceau est ralenti, mais il est plus chaud. Ce sont ces deux aspects que caractérisent les sections efficaces de transfert de quantité de mouvement et d'énergie. Dans le cas présent, le taux de variation de l'énergie thermique d'une population $i$ de masse $m_{i}$ entrant en collision avec une population $n$ de masse $m_{n}$ s'exprime, dans le référentiel du centre de masse :

$\frac{\delta E_{i n}}{\delta t}=v_{i n}^{E} n_{n} \frac{1}{2} \mu v^{2}$

où $\mu$ est la masse réduite, $v$ la vitesse différentielle entre les deux espèces et $v_{\text {in }}^{E}$ la fréquence de collision de transfert d'énergie, calculée à partir de la section efficace de transfert d'énergie $\sigma_{i n}^{E}$ introduite au chapitre $I V$. Or dans ce chapitre, nous avons montré qu'il existe une relation simple entre section efficace de transfert de quantité de mouvement et section efficace de transfert d'énergie. En l'appliquant dans l'équation $V I-32$, nous arrivons à l'expression suivante :

$\frac{\delta E_{\text {in }}}{\delta t}=v_{i n} n_{n} \frac{m_{i} m_{n}}{m_{i}+m_{n}}\left|\overrightarrow{u_{n}}-\overrightarrow{u_{i}}\right|^{2}$

$V I-33$

où $v_{i n}$ est la fréquence de collision de transfert de quantité de mouvement, $\overrightarrow{u_{i}}$ et $\overrightarrow{u_{n}}$ étant les vitesses moyennes des deux espèces. Ce terme correspond à la seconde contribution de l'échange thermique et s'apparente donc à une propriété dispersive des collisions ; c'est un effet de friction.

En général, les vitesses différentielles entre les espèces présentes dans le plasma ionosphérique sont faibles, à l'exception toutefois des espèces légères minoritaires comme $\mathrm{H}^{+}$qui n'apportent cependant qu'une contribution mineure au bilan d'énergie. Cependant, deux configurations particulières peuvent rendre cette contribution importante aux hautes latitudes. 
En effet, nous avons vu qu'au-dessus de $120 \mathrm{~km}$, les ions sont gelés dans le champ magnétique, de telle sorte que leur mouvement est différentié par rapport à celui de l'atmosphère neutre. Si un vent neutre souffle, les ions ne peuvent pas suivre et une vitesse différentielle apparaît qui, dans les régions polaires, peut atteindre des valeurs importantes $\left(\sim 1000 \mathrm{~m} \mathrm{~s}^{-1}\right)$. D'autre part, si l'atmosphère neutre est au repos et qu'un champ de convection est présent dans l'ionosphère, les ions sont mis en mouvement à la vitesse de dérive définie en VI-31. Ces deux effets peuvent apparaître simultanément et ainsi se compenser ou s'amplifier. On a donc une grande variabilité de situations possibles, qui sont étroitement liées à la dynamique de l'atmosphère et de l'ionosphère.

Ces mouvements d'ensemble résultent donc en un chauffage du plasma au travers de l'effet dispersif que nous venons de décrire et comme cet échauffement est local, nous pouvons considérer que du point de vue échange thermique il entraîne un accroissement de la température neutre d'une quantité :

$\delta T_{n}=\frac{m_{n}}{3 k_{b}}\left|\overrightarrow{u_{n}}-\overrightarrow{u_{i}}\right|^{2}$

qui modifie alors le terme d'échange thermique :

$\frac{\delta E_{i n}}{\delta t}=3 k_{b} \sum_{n} n_{n} v_{i n} \frac{m_{i}}{m_{i}+m_{n}}\left(T_{n}+\delta T_{n}-T_{i}\right)$

Avec cette représentation, la description que nous avons faite au chapitre $V$ reste valable car, même si les vitesses sont importantes, les mécanismes locaux d'échange d'énergie restent prépondérants.

En fin de compte, l'effet de la friction fait que les neutres apparaissent plus chauds qu'ils ne sont aux ions. Comme dans la basse ionosphère, ils sont thermalisés par ces neutres, la friction leur impose une température supérieure à celle de l'atmosphère neutre de la quantité $\delta T_{n}$.

Si l'origine de la friction est le champ électrique, en supposant que l'atmosphère est au repos et qu'elle se réduit à sa composante majoritaire de masse $m_{n}$, on peut écrire :

$\delta T_{n}=\frac{m_{n}}{3 k_{b}}\left(\frac{E_{\perp}}{B}\right)^{2}$

soit dans les conditions terrestres un accroissement de température exprimé en Kelvin :

$\delta T_{n}=1,610^{-2} m_{n} E_{\perp}^{2}$

où $m_{n}$ est la masse en amu et $E_{\perp}$ le champ électrique en $\mathrm{mV} \mathrm{m}^{-1}$. Ainsi, au-dessus de $200 \mathrm{~km}$ où l'oxygène est le neutre majoritaire, un champ électrique de $25 \mathrm{mV} \mathrm{m}^{-1}$ entraîne un accroissement de la température ionique de $160 \mathrm{~K}$ par rapport à la température neutre, un champ de $50 \mathrm{mV} \mathrm{m}^{-1}$ un accroissement de $650 \mathrm{~K}$ et un champ de $100 \mathrm{mV} \mathrm{m}^{-1}$, qui n'est pas rare en région aurorale, un accroissement de $2600 \mathrm{~K}$. 
Ceci n'est pas sans conséquence pour l'ionosphère. En effet, cet accroissement de température résulte de la dérive perpendiculaire à la ligne de champ magnétique et crée donc une anisotropie de comportement du plasma ionosphérique. La conséquence de cette anisotropie est une population ionique qui a une fonction de distribution fortement non maxwellienne avec notamment une température dans la direction parallèle au champ différente de celle dans la direction perpendiculaire.

Par ailleurs, la réaction $I V-55$ entre $\mathrm{O}^{+}$et $\mathrm{N}_{2}$, qui conduit à la formation de $\mathrm{NO}^{+}$, l'ion majoritaire de la région $F 1$, est très sensible au niveau d'excitation de la molécule d'azote. Or plus la température est élevée, plus la molécule est excitée et donc réactive, et le taux de cette réaction est donc extrêmement sensible à l'effet de friction (voir annexe $I I$ ). La figure $V I-16$ représente l'évolution du taux de la réaction en fonction de la température de réaction $T_{r}=\frac{T_{i}+T_{n}}{2}$.

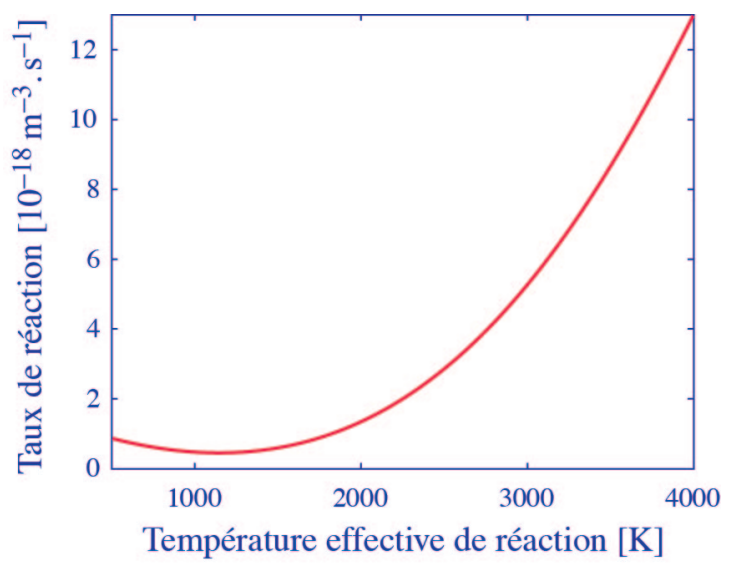

Figure VI-16 : évolution avec la température effective de réaction du taux de la réaction entre $\mathrm{O}^{+}$et $\mathrm{N}_{2}$ qui conduit à la formation de $\mathrm{NO}^{+}$.

On note un fort accroissement du taux de cette réaction avec la température effective de réaction: ce taux croît d'un facteur proche de 25 entre sa valeur minimale (autour de $1000 \mathrm{~K}$ ) et sa valeur à $4000 \mathrm{~K}$. Si l'on considère que l'on a $T_{i}=T_{n}+\delta T_{n}$, l'impact de la friction est considérable puisqu'on a $T_{r}=T_{n}+\frac{\delta T_{n}}{2}$ et la valeur à $4000 \mathrm{~K}$ correspond alors à un champ électrique de $150 \mathrm{mV} \mathrm{m}^{-1}$ pour une température neutre de $1000 \mathrm{~K}$. Lorsque le taux croît, les ions $\mathrm{O}^{+}$ont une durée de vie chimique qui se réduit vis à vis de cette réaction et se transforment rapidement en ions $\mathrm{NO}^{+}$beaucoup plus lourds. Ces derniers deviennent donc majoritaires jusqu'à plus haute altitude et déplacent la zone de transition entre ions moléculaires et ions atomiques vers les altitudes supérieures. La figure VI-17 montre l'évolution du profil de composition ionique $p=\frac{\mathrm{n}\left(\mathrm{NO}^{+}\right)}{n_{e}}$ au cours d'une perturbation de champ électrique. Les courbes extrêmes montrent la composition avant (courbe de gauche) et après (courbe de droite) l'application d'un champ électrique d'une amplitude évaluée à $65 \mathrm{mV} \mathrm{m}^{-1}$. 
On constate l'effet important du champ sur la composition puisque l'altitude de transition $(p=0,5)$ croît depuis $215 \mathrm{~km}$ en période sans champ jusqu'à $255 \mathrm{~km}$ lors du maximum de la perturbation.

21:55

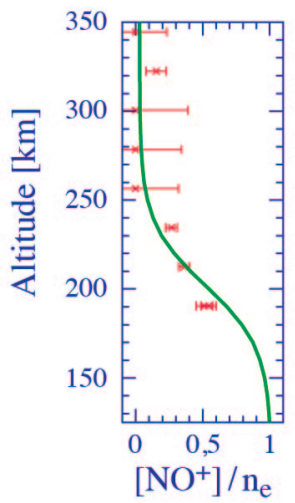

22:13

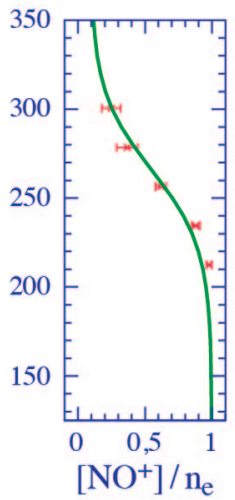

23:00

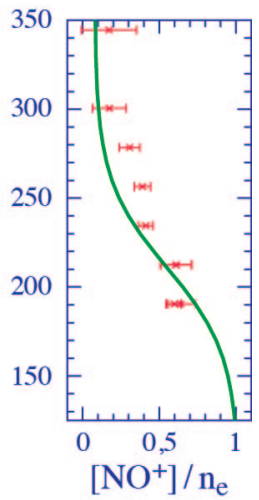

Figure VI-17 : comparaison entre la composition mesurée (croix) et une simulation numérique (ligne pleine) à trois instants différents qui encadrent une perturbation de champ électrique : à 21:55 et 23:00 TU champ électrique faible et maximum de $65 \mathrm{mV} \mathrm{m}^{-1}$ à $22: 13 \mathrm{TU}$

L'effet du champ électrique est quasi-immédiat sur la composition ionosphérique mais il n'est sensible réellement que si le champ dépasse $30 \mathrm{mV} \mathrm{m}^{-1}\left(\delta T_{n}=250 \mathrm{~K}\right)$.

\section{ii. Chauffage électronique par instabilité}

Les électrons sont beaucoup plus légers que les ions et ne sont pas sensibles à cet effet de friction. Par contre, ils sont piégés autour des lignes de champ dès $90 \mathrm{~km}$ environ et il est alors possible d'observer un mouvement différentiel entre ions et électrons en présence du champ électrique, qui est interprétable comme un courant dans l'ionosphère.

L'apparition de ce courant est susceptible d'engendrer une instabilité, dite instabilité à deux faisceaux, qui est due au déplacement relatif entre les fonctions de distributions ionique et électronique dans l'espace des phases. Cette instabilité est l'instabilité de Farley-Buneman et a pour effet d'exciter des ondes électrostatiques dans la direction du courant. Cette instabilité croît en présence du courant puis sature en entraînant un échauffement des électrons qui, du fait du rapport de masse entre ions et électrons, absorbent la majeure partie de l'énergie associée à l'instabilité.

Pour que cette instabilité soit mise en œuvre, il faut un courant important et donc un grand champ de convection. En région aurorale, ce mécanisme est perceptible pour des champs supérieurs à $50 \mathrm{mV} \mathrm{m}^{-1}$ et présente un effet maximal vers $110-120 \mathrm{~km}$.

On peut voir cette interaction entre électron et onde électrostatique comme une collision entre un électron et une particule virtuelle, le plasmon, qui transporte l'énergie de l'instabilité. On peut dès lors modéliser cette interaction par une fréquence de collision 
anormale $\mathrm{v}_{e}{ }^{*}$; cette fréquence de collision peut devenir prépondérante devant les autres fréquences de collision et atteindre une fraction non négligeable de la gyrofréquence électronique (10 à $30 \%)$. En utilisant cette approche collisionnelle, on peut alors introduire le terme de chauffage associé à l'instabilité dans l'équation de l'énergie des électrons :

$Q_{e}^{*}=\mathrm{v}_{e}^{*} n_{e} m_{e}\left(\mathrm{v}_{d}-c_{i}\right)^{2}$

$V I-38$

où $v_{d}$ est l'amplitude de la vitesse différentielle entre les ions et les électrons et $c_{i}$ est la vitesse acoustique des ions :

$c_{i}^{2}=\frac{k_{b}\left(T_{i}+T_{e}\right)}{m_{i}}$

$V I-39$

\section{b. L'action du courant aligné}

Ainsi que nous l'avons décrit dans le chapitre $I I I$, le couplage électrodynamique fait intervenir des courants de particules alignés au lignes de champ et relient l'ionosphère à la magnétosphère. Ces courants ont un effet direct sur le comportement des électrons.

La formule $V-178$ qui donne l'expression du flux de chaleur électronique, met en évidence l'effet thermoélectrique auxquels les électrons sont soumis lorsqu'on applique un courant. Le rapport entre la conduction thermique et l'effet thermoélectrique est tel que pour une température de $3000 \mathrm{~K}$ et un gradient de $0,3 \mathrm{~K} \mathrm{~km}^{-1}$ (ce que l'on observe vers $1000 \mathrm{~km}$ ), un courant aligné de $1 \mu \mathrm{A} \mathrm{m}^{-2}$ apporte une contribution au flux de chaleur qui est environ 2500 fois plus importante que la conduction thermique. Comme la divergence du flux intervient dans le bilan énergétique, on a donc une forte sensibilité de la température électronique vis à vis d'un courant aligné. Ainsi, si nous nous plaçons dans les régions aurorales où nous pouvons projeter les équations le long de la verticale (en supposant que le champ magnétique est vertical), nous pouvons écrire au-dessus de la région $F$ la contribution de l'effet thermoélectrique sous la forme :

$q_{e}^{t h} \approx \frac{k_{b} T_{e}}{e} J_{/ /}$

en notant $J_{/ /}$la composante du courant aligné. Or aux altitudes auxquelles on se place, on peut considérer que le courant a une divergence nulle qui résulte du piégeage des particules par le champ magnétique. Nous avons donc :

$\frac{d J_{\|}}{d z}=0$

La contribution de cet effet thermoélectrique au bilan énergétique des électrons devient alors :

$Q=\frac{2}{3} \frac{1}{k_{b} n_{e}} \frac{d q_{e}^{t h}}{d z}=\frac{2}{3} \frac{J_{\|}}{e n_{e}} \frac{d T_{e}}{d z}$

En considérant une situation initiale où la température croît avec l'altitude, un courant positif donne une contribution positive au bilan d'énergie et agit donc comme une source de chaleur pour les électrons : la température va donc croître sous l'effet du courant. 
Un courant positif correspond à un mouvement différentiel positif entre ions et électrons, qui résulte généralement de précipitations électroniques d'origine magnétosphérique. Ces précipitations agissent sur la composante thermoélectrique mais également sur la conduction thermique au travers des interactions entre les électrons thermiques et les électrons suprathermiques de basse énergie. Il y a donc un effet cumulatif.

\section{UN EXEMPLE DE PERTURBATION ÉLECTRODYNAMIQUE}

La figure $V I-18$ représente un sondage effectué par les radars EISCAT (voir annexe III) dans l'après-midi et dans la soirée du 24 mars 1995. Les radars UHF et VHF fonctionnaient simultanément pendant cette campagne, le radar UHF ayant un axe de visée aligné le long de la direction du champ magnétique et la radar VHF ayant un axe de visée vertical.

Nous avons reporté les résultats du sondage pour l'UHF (panneau du haut) et le VHF (panneau du bas). Pour chacun de radars, nous avons représenté les séquences temporelles de la concentration électronique ( $\mathrm{Ne}$ ), de la température électronique (Te), de la température ionique (Ti) et de la vitesse ionique le long de la ligne de visée (Vi) pour toutes les altitudes sondées. Enfin, le radar UHF était utilisé en mode tristatique (voir annexe III) et nous avons donc pu reconstituer les vecteurs vitesse ionique et estimer le champ électrique de convection que nous avons reporté sur la figure couleur (composante nord en rouge et est en vert). Nous n'allons pas discuter en détail les observations et nous allons nous concentrer sur quelques aspects.

Durant l'après-midi, jusque vers 19:00 TU, l'activité magnétique est faible et le champ électrique quasiment nul. L'ionosphère que l'on observe est alors caractéristique d'une ionosphère diurne. En particulier, on constate une décroissance de la concentration électronique avec le temps (on est à midi local vers 11:00 TU). Comme nous sommes en hiver, l'angle solaire zénithal est très élevé $\left(\chi=80^{\circ}\right.$ à $15: 00 \mathrm{TU}$ et $\chi=110^{\circ}$ à 23:00 TU). On remarque que la couche de la région $F$ croît en altitude avec le temps suite à l'accroissement de $\chi$ et que la région $E$ disparaît (vers 18:00 TU, on ne mesure plus de concentration électronique à $100 \mathrm{~km}$ avec l'UHF). Cette décroissance de la concentration s'accompagne d'une décroissance de la température électronique (panneau du haut) qui résulte de l'amoindrissement du chauffage électronique par les photoélectrons. A haute altitude, la température est toujours élevée, tandis qu'à basse altitude, les collisions avec les ions et les neutres contribuent à thermaliser les électrons. De fait, on observe la même tendance pour les ions, puisque ceuxci sont principalement chauffés par les électrons.

Après 19:30 TU, nous entrons dans l'ovale auroral et l'ionosphère est soumise à de fortes perturbations bien visibles sur tous les paramètres mesurés. La différence de visée entre le VHF et l'UHF montre bien que ces perturbations sont alignées aux lignes de champ magnétique : le radar VHF "voit" l'ionosphère plus au nord par rapport aux lignes de champs dont les pieds se situent au voisinage des radars, alors que l'UHF sonde ces lignes de champ sur plus de $500 \mathrm{~km}$. Nous décrirons donc uniquement les résultats issus de l'UHF. 

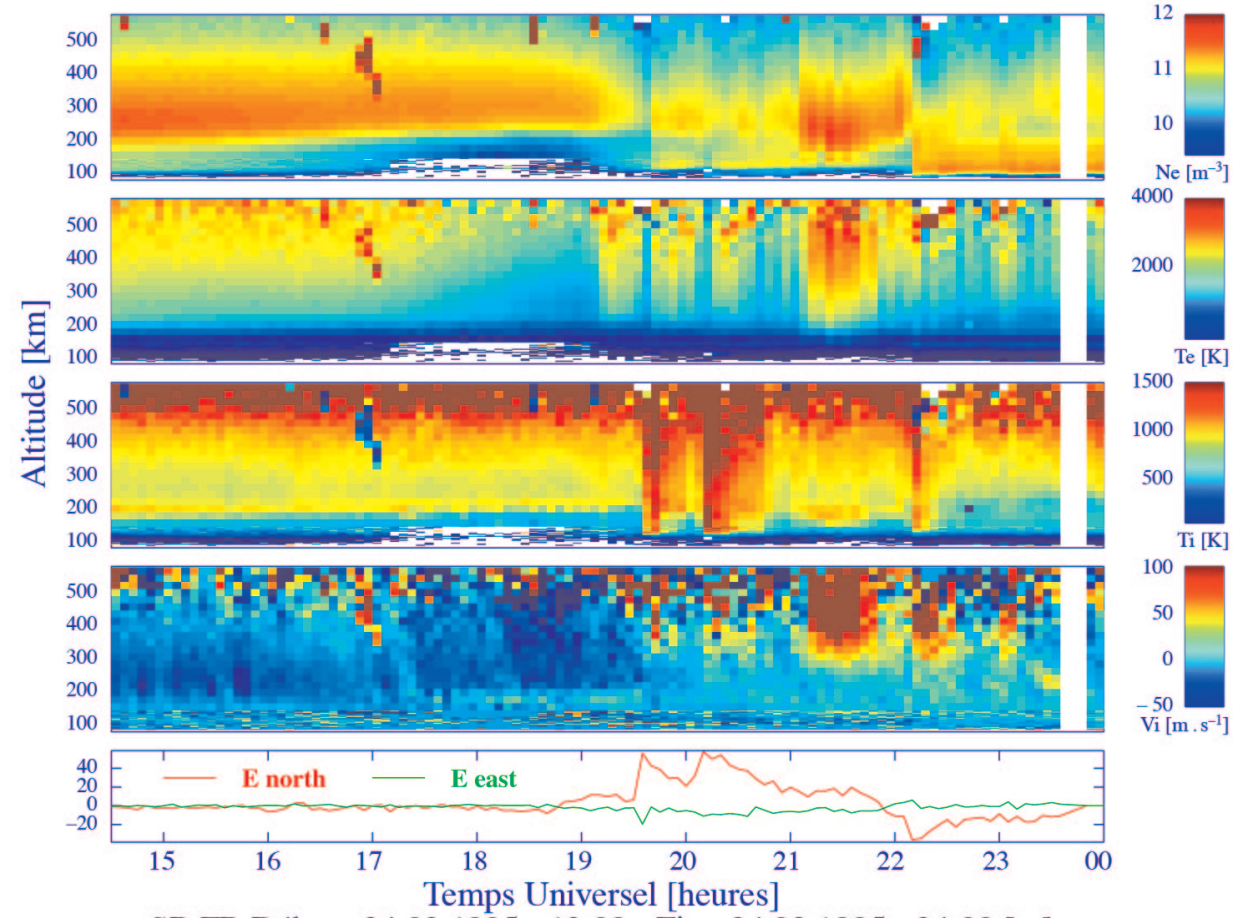

SP-FR Début : 24.03.1995 - 13:00 - Fin : 24.03.1995 - 24:00 [ut]

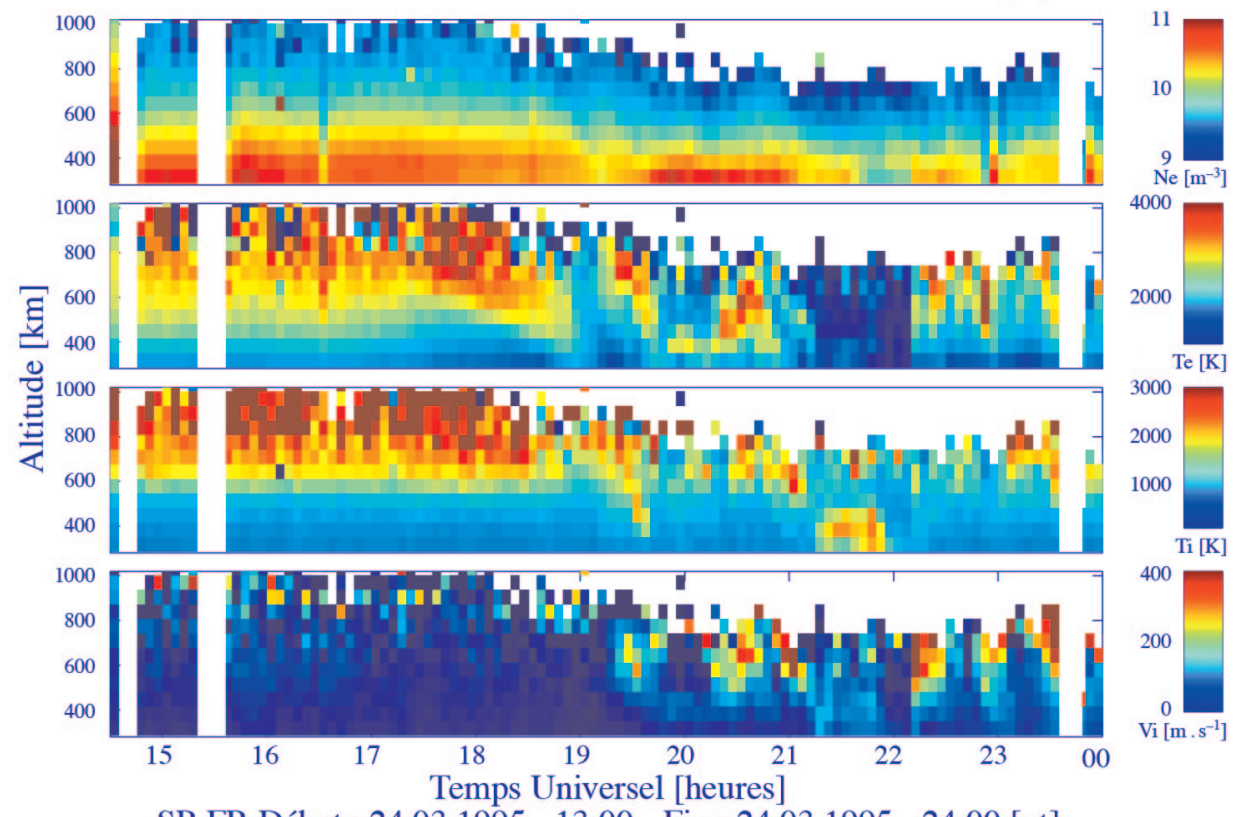

SP-FR Début : 24.03.1995 - 13:00 - Fin : 24.03.1995 - 24:00 [ut]

Figure VI-18: tracés temporels des mesures des paramètres plasmas ionosphériques $\left(n_{e}, T_{e}, T_{i}\right.$ et $\left.v_{i}\right)$ effectuées par les radars UHF et VHF d'EISCAT le 24 mars 1995 en région aurorale 
Avant que le champ électrique ne commence à croître, on observe des précipitations électroniques qui produisent des électrons entre 120 et $150 \mathrm{~km}$ et chauffent les électrons thermiques à haute altitude. Il s'agit certainement d'un effet de conduction de chaleur qui fait croître le flux de chaleur à l'altitude supérieure et donc également la température. Après cet événement, on en observe un second vers 19:45 TU au cours duquel le champ électrique croît fortement. L'énergie des particules précipitant a changé puisque l'on a une production électronique entre 200 et $300 \mathrm{~km}$, caractéristique de la présence d'électrons de basse énergie. Ceci entraîne un échauffement de la température électronique et l'effet de friction associé à la convection fait croître la température ionique. On constate un faible accroissement de la vitesse ionique, qui laisse supposer la présence d'un courant aligné faible ; l'accroissement de la température électronique pourrait être associé à ce courant. Entre 20:00 et 21:00 TU, on observe une autre perturbation qui produit les mêmes effets. Entre 21:00 et 22:00 TU, l'énergie des particules précipitant change considérablement et alors que l'on a un champ électrique constant, on observe une impulsion de vitesses élevées ; ceci suppose la présence d'un courant aligné qui chauffe les températures électroniques. Après cet événement, le champ électrique décroît en même temps que l'énergie moyenne des électrons précipitants, ainsi que l'atteste la remontée de la couche d'électrons créés. Le profil de composition à 21:55 TU de la figure VI-17 correspond à cette période. Après 22:00 TU, le champ électrique s'inverse en même temps que la direction de la convection (elle était orientée Ouest-Est avant le changement de direction). Les énergies des électrons qui précipitent deviennent beaucoup plus élevées, puisqu'on a une production vers $100 \mathrm{~km}$. L'inversion de champ électrique est associée à un échappement d'ions mais pas à un accroissement significatif de la température électronique. Un chauffage ionique est associé à cet événement de champ électrique : le profil à 22:13 TU de la figure VI-17 montre l'effet de ce champ sur la composition ionosphérique. Après cet événement, le champ électrique décroît et seules des précipitations se maintiennent à des niveaux d'énergie élevée. On observe une production d'électrons à plus haute altitude (et donc plus basse énergie) qui indique un spectre de précipitations à deux sous-populations. La sous-population basse énergie s'intensifie vers 24:00 TU; le profil à 23:00 TU de la figure $V I-17$ représente cette phase d'équilibre où l'ionosphère est seulement soumise à des précipitations.

En conclusion de cette description, nous retiendrons essentiellement la formidable dynamique de l'ionosphère dans son couplage électrodynamique avec la magnétosphère. Les phases de production d'ions et de chauffage ionique ou électronique se succèdent avec un rythme qui peut être élevé ; dès que ces actions cessent, l'ionosphère rétablit très rapidement (quelques dizaines de secondes) un équilibre sur les grandeurs énergétiques, mais le recouvrement sur les particules ionosphériques peut prendre beaucoup plus de temps (quelques dizaines de minutes). Les précipitations dont nous voyons la trace sur ces panneaux couleur sont également visibles à l'œil nu puisqu'elles sont la cause des aurores que nous discuterons au chapitre VII. 


\section{COUPLAGE IONOSPHÈRE/THERMOSPHÈRE : DIFFUSION AMBIPOLAIRE DANS UN CHAMP DE GRAVITÉ}

Nous avons décrit au chapitre $V$ le mécanisme de diffusion de l'ion $\mathrm{O}^{+}$dans la région $F$ (équation $V-96$ ), où il est l'ion majoritaire. Il est évident que le principe peut être étendu à tout ion de l'ionosphère et même à un ion qui serait une moyenne de toutes les espèces ioniques présentes. De plus, les équations ont été écrites en projection le long de la verticale, mais il est clair que toute projection dans une direction qui fait un angle fixe par rapport à la verticale s'en déduit immédiatement. Ceci s'applique en particulier en région aurorale où le mouvement s'organise le long des lignes de champ, inclinées par rapport à la verticale. Si l'on note $I$ l'angle magnétique qui correspond à l'angle entre la direction du champ et l'horizontale, on peut récrire facilement l'équation de diffusion de l'ion majoritaire le long de la ligne de champ. En notant $v_{i / /}(v$ minuscule) la vitesse d'un ion le long de la ligne de champ dans le repère du vent neutre, on peut donc écrire l'équation suivante :

$v_{i / l}=-\frac{1}{m_{i} v_{i n}}\left(m_{i} g \sin I+\frac{1}{n_{e}} \nabla_{/ /}\left\{n_{e} k_{b}\left(T_{e}+T_{i}\right)\right\}\right)$

où $\nabla_{/ /}$exprime le gradient le long de la ligne de champ. Par rapport à l'équation $V-96$, nous considérons que la température varie avec l'altitude. Notons conventionnellement $T_{p}=T_{i}+T_{e}$ la température plasma. Si on note $s$ l'abscisse curviligne le long de cette ligne de champ, nous avons $d z=\sin I d s$ et nous pouvons donc exprimer le gradient par rapport à l'altitude. Si de plus, nous nous intéressons au mouvement moyen de l'ionosphère, nous pouvons écrire une équation de diffusion pour un ion moyen :

$v_{i / /}=-\frac{\sin I}{\left.<m_{i} v_{i n}\right\rangle}\left(<m_{i}>g+\frac{1}{n_{e}} \nabla_{/ /}\left(n_{e} k_{b} T_{p}\right)\right)$

$V I-44$

où les termes entre crochets représentent des moyennes faites sur les différents constituants ioniques. On utilise généralement la vitesse de diffusion ambipolaire $v_{\text {diff }}$ définie, le long de la verticale, par :

$v_{\text {diff }}=\frac{v_{\text {i/l }}}{\sin I}$

$V I-45$

Cette vitesse de diffusion représente la vitesse à laquelle les ions diffusent verticalement dans un gaz neutre immobile. Si on développe la dérivée de l'équation $V I-44$, on arrive facilement à l'expression :

$v_{i / /}=-\frac{k_{b} T_{p}}{<m_{i} v_{i n}>}\left(\frac{<m_{i}>g}{k_{b} T_{p}}+\frac{1 d n_{e}}{n_{e} d z}+\frac{1 d T_{p}}{T_{p} d z}\right)$

Cette vitesse de diffusion néglige la contribution de la diffusion thermique (voir dans l'annexe $I$ termes en flux de chaleur dans les collisions de l'équation d'impulsion). si on note $\lambda$ le coefficient de diffusion thermique et si on introduit la température réduite $T_{r}=\frac{T_{n}+T_{i}}{2}$, qui est telle que le rapport $\frac{T_{i}}{T_{r}}$ vaut 1 quand $T_{i}=T_{n}$, l'équation de diffusion devient alors : 
$v_{i / /}=-D_{a} \frac{T_{i}}{T_{r}}\left(\frac{<m_{i}>g}{k_{b} T_{p}}+\frac{1 d n_{e}}{n_{e} d z}+\frac{1 d T_{p}}{T_{p} d z}+\frac{\lambda d T_{p}}{T_{p} d z}\right)$

$D_{a}$ est le coefficient de diffusion ambipolaire que nous avons déjà introduit au chapitre $V$ (équation $V-94$ ), mais appliqué à un l'ion moyen, soit :

$D_{a}=\frac{k_{b} T_{p}}{<m_{i} v_{i n}>}$

$V I-48$

Cette expression de la vitesse de diffusion est très intéressante, puisque la mesure de $n_{e}, T_{e}$ et $T_{i}$ suffit à la calculer. Or, cette mesure se fait à partir de radars géophysiques. Dans le paragraphe suivant, nous montrerons comment la connaissance de la vitesse de diffusion ambipolaire permet, sous certaines conditions, d'accéder au vent neutre le long d'un méridien géomagnétique à partir de la mesure faite par des radars.

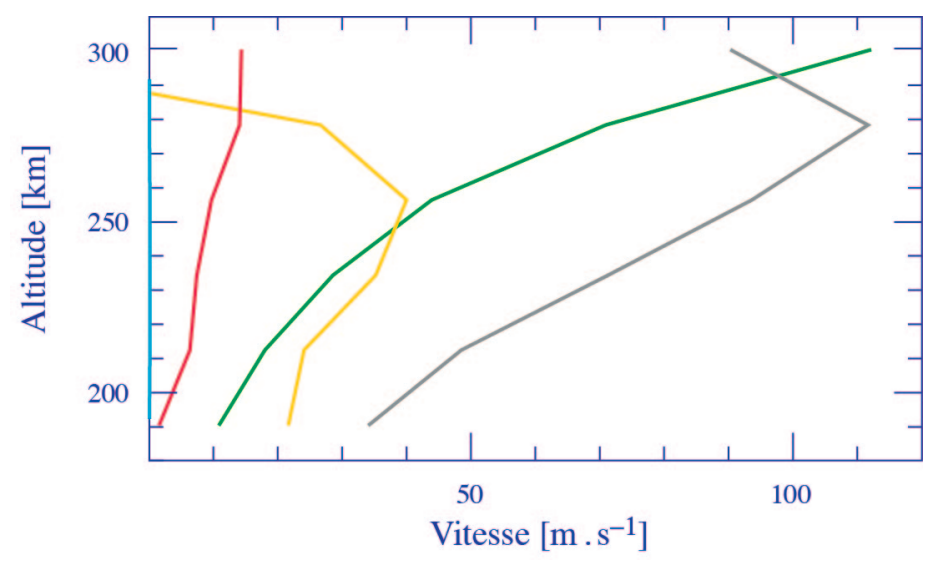

Figure $V I-19$ : les différentes contribution à la vitesse de diffusion donnée en $V I-47$

En rouge, nous avons le terme dû à la dérivée de la température de plasma. En jaune, celui qui porte sur la dérivée de la densité électronique. En vert, nous voyons le terme de gravité. Le terme dû au coefficient de diffusion thermique (bleu) est négligeable. Le trait gris représente la somme.

La figure VI-19 montre la moyenne de la vitesse de diffusion et de ses différentes composantes sur cinq journées consécutives de mesure, en janvier 1993, à partir du radar EISCAT situé dans l'ovale auroral. L'indice décimétrique $\mathrm{f}_{10.7}$ varie entre 100 et 110 , et l'indice magnétique Ap varie entre 4 et 14 pour ces cinq jours. Nous sommes donc dans des conditions ionosphériques calmes. Sur cette figure, nous constatons que le terme de diffusion thermique joue un rôle négligeable dans l'expression de la diffusion ambipolaire et donc que l'équation $V I-46$ peut être utilisée à la place de l'équation $V I-47$ sans grande erreur. Les termes de gravité et de gradient de densité électronique sont les contributions prépondérantes. En particulier, le dernier terme, qui est l'inverse de la hauteur d'échelle du plasma (voir chapitre $V$ ), voit son importance varier avec l'altitude et peut devenir très important dans les régions à forte variation de concentration électronique. La diffusion ambipolaire, au cours de cette expérience, est orientée toujours positivement, ce qui correspond à un mou- 
vement vers le nord. Les amplitudes peuvent paraître élevées, mais il faut prendre garde qu'il s'agit d'une projection : il faut diviser par 4 en cet endroit de haute latitude pour obtenir la vitesse le long de la ligne de force (équation VI-61). Dans ce cas, la vitesse positive correspond à une diffusion vers le bas.

\section{COUPLAGE IONOSPHÈRE-MAGNÉTOSPHÈRE : LE VENT POLAIRE}

Nous avons vu dans le chapitre $V$ que l'ion minoritaire $\mathrm{H}^{+}$diffuse vers les hautes altitudes sous l'action du champ de polarisation induit par la séparation de charges entre les électrons et l'ion majoritaire $\mathrm{O}^{+}$. Nous allons nous intéresser dans ce paragraphe à l'échappement qui peut résulter de cette diffusion. Le mécanisme d'échappement concerne principalement des ions légers comme $\mathrm{H}^{+}$et $\mathrm{He}^{+}$et est de nature très différente de celui qui conduit à l'évaporation thermique discutée au chapitre $I I$. En fait, ce mécanisme s'apparente à celui qui donne naissance au vent solaire (voir chapitre $I$ ), et par analogie on l'appelle vent polaire. Dans une ionosphère magnétisée, le vent polaire ne peut se développer qu'aux très hautes latitudes, près des pôles magnétiques, dans les régions où les lignes de champ magnétique englobent un volume suffisamment grand pour que le phénomène perdure ; dans la plasmasphère, le vent polaire ne peut être qu'un phénomène transitoire, car l'équilibre de pression dans les tubes de champ y est atteint très rapidement en comparaison des régions des hautes latitudes. Ce phénomène est d'autant plus stable que les lignes de champs sont étirées, et il atteint un paroxysme le long des lignes de champs reconnectées au champ magnétique interplanétaire car les ions sont alors éjectés vers le milieu interplanétaire. En fait le dénominatif vent polaire concerne plus généralement le processus d'extraction des ions de l'ionosphère des hautes latitudes et s'applique à tous les ions. Les mécanismes d'extraction peuvent être de natures diverses et notamment correspondre à des interactions entre ondes électromagnétiques et particules chargées (ions). Nous étudierons ici le vent polaire d'origine thermique dont la seule source d'accélération est le champ de polarisation du plasma, qui n'agit donc que sur les espèces légères et minoritaires de l'ionosphère.

Nous nous placerons dans les mêmes conditions que celles du chapitre $V$, à savoir à haute latitude au-dessus de la région $F$, et notamment nous ne considérerons que les projections des équations le long des lignes de champ magnétique que nous supposerons verticales (d'après le paragraphe précédent, nous pouvons dire que c'est vrai à un facteur sin $I$ près). En considérant que l'état d'équilibre est atteint et que l'ion majoritaire (en l'occurrence $\mathrm{O}^{+}$ référence 1 ) est en équilibre hydrostatique, nous pouvons écrire l'équation d'impulsion de l'ion $\mathrm{H}^{+}$(référencé 2) sous la forme :

$n_{2} m_{2} u_{2} \frac{d u_{2}}{d z}+\frac{d}{d z}\left(n_{2} k_{b} T_{2}\right)+n_{2} m_{2} g-n_{2} e E=-v_{2 l} n_{2} m_{2} u_{2}$

où $\mathrm{v}_{2 l}$ est la fréquence de collision entre l'ion $\mathrm{H}^{+}$et l'ion $\mathrm{O}^{+}$et $E$ est le champ de polarisation le long de cette verticale dont nous rappelons l'expression :

$e E=-\frac{k_{b} T_{e}}{n_{e}} \frac{d n_{e}}{d z}=m_{1} \frac{T_{e}}{T_{e}+T_{l}} g$ 
en supposant que $\mathrm{O}^{+}$et les électrons ont des températures constantes avec l'altitude. L'hypothèse d'une température constante est assez bien adaptée à la condition de l'équilibre hydrostatique car seule la conduction thermique intervient. Cependant, lorsqu'une espèce a une vitesse d'écoulement importante, comme c'est le cas pour l'ion $\mathrm{H}^{+}$dans le vent polaire, le phénomène de diffusion est susceptible de devenir prépondérant par rapport à la conduction et l'hypothèse d'une température constante peut être remise en question. Pour prendre en compte une variation éventuelle de la température avec l'altitude, sans compliquer les calculs outre mesure, nous supposerons alors que l'ion $\mathrm{H}^{+}$vérifie une équation d'état pseudo adiabatique de la forme :

$n_{2}{ }^{1-\gamma} T_{2}=$ constante $\quad \Rightarrow \quad(1-\gamma) \frac{d n_{2}}{n_{2}}+\frac{d T_{2}}{T_{2}}=0$

$V I-51$

où $\gamma$ est un paramètre supérieur à 1 (il s'apparente au rapport des chaleurs spécifiques); la condition d'une température $T_{2}$ constante peut être traduite par $\gamma=1$. Cette équation d'état s'ajoute à l'équation de conservation de l'ion $\mathrm{H}^{+}$:

$n_{2} u_{2}=$ constante $\quad \Rightarrow \quad \frac{d n_{2}}{n_{2}}+\frac{d u_{2}}{u_{2}}=0$

pour donner la condition équivalente suivante :

$u_{2}^{\gamma-1} T_{2}=$ constante $\quad \Rightarrow \quad(\gamma-1) \frac{d u_{2}}{u_{2}}+\frac{d T_{2}}{T_{2}}=0$

$V I-53$

En utilisant la masse effective $m_{2}{ }^{\prime}$ de l'ion $\mathrm{H}^{+}$définie en $V-117$, qui inclut l'accélération due au champ de polarisation, on peut récrire $V I-49$ sous la forme :

$n_{2} m_{2} u_{2}^{2} \frac{1}{u_{2}} \frac{d u_{2}}{d z}+n_{2} k_{b} T_{2}\left(\frac{1}{n_{2}} \frac{d n_{2}}{d z}+\frac{1}{T_{2}} \frac{d T_{2}}{d z}\right)+n_{2} m_{2}^{\prime} g=-v_{21} n_{2} m_{2} u_{2}$

ou :

$n_{2} m_{2} u_{2}^{2} \frac{1}{u_{2}} \frac{d u_{2}}{d z}+n_{2} k_{b} T_{2}\left(-\frac{1}{u_{2}} \frac{d u_{2}}{d z}+(1-\gamma) \frac{1}{u_{2}} \frac{d u_{2}}{d z}\right)+n_{2} m_{2}^{\prime} g=-v_{21} n_{2} m_{2} u_{2}$

Pour simplifier cette équation, nous introduisons alors la vitesse du son $c_{2}$ de l'ion $\mathrm{H}^{+}$: $c_{2}{ }^{2}=\gamma \frac{k_{b} T_{2}}{m_{2}}$, et nous définissons le nombre de Mach $M_{2}=\frac{u_{2}}{c_{2}}$. Ce nombre vérifie la propriété suivante :

$$
\left.\begin{array}{rl}
\frac{d M_{2}}{M_{2}} & =\frac{d u_{2}}{u_{2}}-\frac{1}{2} \frac{d T_{2}}{T_{2}} \\
& =\frac{\gamma+1}{2} \frac{d u_{2}}{u_{2}}
\end{array}\right\} \Rightarrow M_{2}^{2} u_{2}^{-(\gamma+1)}=\text { constante }
$$

et varie donc dans le même sens que la vitesse. En divisant l'équation VI-55 par $n_{2} m_{2} c_{2}{ }^{2}$, on trouve alors l'équation d'évolution pour $M_{2}$ :

$\frac{\gamma+1}{2} \frac{M_{2}{ }^{2}-1}{M_{2}} \frac{d M_{2}}{d z}=-\frac{m_{2}{ }^{\prime}}{m_{2}} \frac{g}{c_{2}{ }^{2}}-v_{21} \frac{M_{2}}{c_{2}}$ 
L'équation ainsi obtenue s'apparente à l'équation de la tuyère de Laval ; elle définit l'évolution du nombre de Mach le long de la direction d'écoulement, qui correspond ici à la verticale. Cette équation est équivalente à l'équation de diffusion $V-116$, avec cependant une différence concernant l'évolution de la température qui était supposée constante. Toutefois, cette équation est mieux adaptée que l'équation de diffusion pour les écoulements à grandes vitesses qui caractérisent le vent polaire. En effet, si on définit un écoulement subsonique (respectivement supersonique) comme étant un écoulement de nombre de Mach $M_{2}<1$ (respectivement $M_{2}>1$ ), l'équation $V I-57$ définit les conditions de transition d'un régime à l'autre et en particulier, elle met en évidence le terme source prépondérant pour le mouvement des ions $\mathrm{H}^{+}$en délimitant les régions d'influence des différentes sources. Dans le modèle que nous avons développé, les termes sources correspondent aux actions du champ de polarisation (compensées par la gravité) et de la friction des ions $\mathrm{H}^{+}$sur les ions $\mathrm{O}^{+}$.

Nous allons discuter les différentes solutions de l'équation VI-57 et en particulier nous allons nous intéresser à la manière dont évolue le nombre de Mach avec l'altitude. La figure VI-20 représente la famille de solutions de cette équation. Nous avons délimité certaines régions et caractérisé les solutions pour faciliter la compréhension du mécanisme mis en jeu. En particulier, les courbes en rouge représentent des écoulements supersoniques, tandis que les courbes en bleu sont les solutions subsoniques.

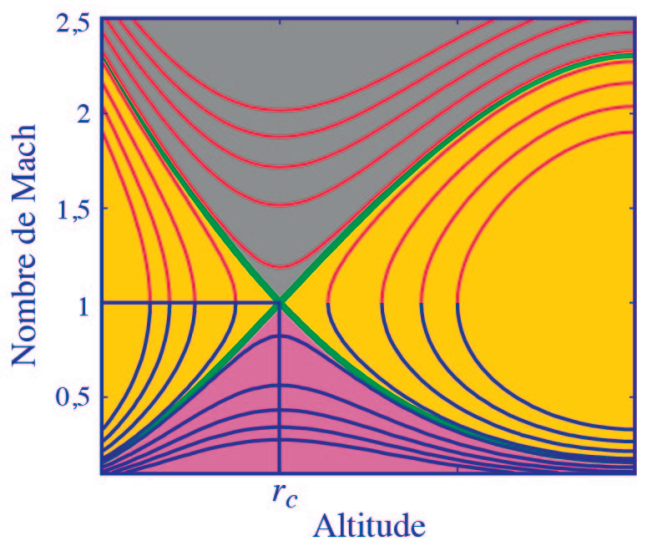

Figure VI-20 : famille de solutions de l'équation $V I-57$ représentées en coordonnées (altitude; nombre de Mach)

Le point critique est repéré par $r_{c}$. Les écoulements supersoniques (subsoniques) sont représentés en rouge (bleu). Les différentes régions sont discutées dans le texte.

Aux basses altitudes, près de la région de production des ions $\mathrm{H}^{+}$, la vitesse d'écoulement est faible de telle sorte que la condition $M_{2}<1$ est vérifiée ; $M_{2}$ décroît alors avec l'altitude si le membre de droite est positif, tandis que $M_{2}$ croît si ce membre est négatif. Or le membre de droite est constitué par la somme des deux sources que nous venons d'identifier et donc, comme ces sources sont de signe opposé, le signe du membre de droite caractérise le terme prépondérant dans l'accélération de l'ion $\mathrm{H}^{+}$. 
L'hypothèse de positivité correspond à :

$-\frac{m_{2}^{\prime}}{m_{2}} g>v_{21} u_{2}$

VI-58

et se traduit donc par une accélération verticale due au champ de polarisation, qui est alors plus importante que la friction de l'ion $\mathrm{H}^{+}$dans l'ion $\mathrm{O}^{+}$. Les ions $\mathrm{H}^{+}$ont donc tendance à s'établir en équilibre hydrostatique car avec un nombre de Mach inférieur à 1 , les processus thermiques dominent; leur nombre de Mach et en conséquence leur vitesse, décroît avec l'altitude. La solution extrême, avec une friction négligeable $\left(M_{2}=0\right)$ est la deuxième solution de l'équation de diffusion du chapitre $V$, qui donne à l'ion $\mathrm{H}^{+}$une hauteur d'échelle négative (correspondant à la masse effective négative $m_{2}{ }^{\prime}$ ). La concentration de l'ion croît avec l'altitude alors que celle de l'ion majoritaire décroît. Cet ion minoritaire devient donc majoritaire à haute altitude et les équations précédentes tombent en défaut dès que l'ion ne peut plus être considéré comme minoritaire.

Sur la figure VI-20, les solutions correspondent aux courbes en bleu dans la région $r>r_{c}$. Etant donné la structure de l'équation et la forme des termes sources, on peut affirmer que si la condition de positivité est réalisée à une certaine altitude, elle l'est à toute altitude supérieure et donc, si le nombre de Mach décroît à une altitude, il décroît au-delà. En effet, la force de friction dépend de la concentration de l'ion majoritaire qui a une hauteur d'échelle beaucoup plus petite que la hauteur d'échelle du terme d'accélération. Par conséquent, si la positivité est assurée à une altitude, elle l'est également à toute altitude supérieure ; la dépendance de la friction vis à vis du nombre de Mach ne fait qu'accroître ce phénomène. De fait, on observe que toutes les solutions décroissent continûment.

L'hypothèse de négativité traduit le fait que le mécanisme de diffusion de l'ion $\mathrm{H}^{+}$dans l'ion $\mathrm{O}^{+}$contrôle le mouvement de l'ion minoritaire. La solution extrême correspond à la première solution de l'équation de diffusion du chapitre $V$ qui donne un ion qui diffuse avec la hauteur d'échelle du plasma. Nous verrons plus loin à quelle courbe correspond cette solution. Dans cette configuration, la vitesse croît donc avec l'altitude dans la région où la friction domine. Cette condition est nécessaire pour l'établissement du vent polaire, mais nous allons voir qu'elle n'est pas suffisante.

Sur la figure $V I-20$, les solutions correspondantes sont les courbes en bleu situées dans la région $r<r_{c}$. Tant que la négativité est assurée, les ions $\mathrm{H}^{+}$peuvent diffuser avec une vitesse croissante, ainsi que le montrent les courbes. Parmi les solutions, on peut distinguer deux classes de courbes. D'une part, on trouve des courbes dans la région rose qui restent avec un nombre de Mach inférieur à 1 et d'autre part, on a des courbes dans la région jaune qui atteignent la valeur $M_{2}=1$. On peut associer ces classes aux deux cas suivants, respectivement :

- à une altitude donnée pour laquelle on a $M<1$, la condition de positivité se réalise ;

- la condition de négativité est réalisée jusqu'à $M=1$.

Nous avons vu que si la condition de positivité se réalise à une altitude, elle est réalisée à toute altitude supérieure. Par conséquent, dans le premier cas, nous pouvons affirmer qu'audessus du point où la condition s'inverse, le nombre de Mach décroît. C'est ce que l'on observe dans la région rose où le nombre de Mach croît pour $r<r_{c}$ et atteint un maximum 
au point critique $r_{c}$ pour décroître au-delà ; le point critique correspond à l'altitude où la friction cède le pas à l'accélération. De fait, on peut généraliser cette propriété à toute la figure et caractériser le point critique comme étant l'altitude où accélération et friction se compensent exactement : les altitudes inférieures à $r_{c}$ correspondent à une région où la friction domine (négativité) et les altitudes supérieures à $r_{c}$ correspondent à une région où l'accélération domine (positivité).

D'après ce que nous venons de discuter, les courbes de la région rose peuvent donc être décrites dans leur totalité et rassemblent toutes les solutions de l'équilibre diffusif présentées au chapitre $V$. En particulier la courbe $M_{2}=0$ correspond à l'équilibre hydrostatique et la courbe en vert qui sépare la région jaune de la région rose pour $r<r_{c}$ correspond à la solution de diffusion à flux limite avec la hauteur d'échelle du plasma. Il apparaît alors que les solutions de la région jaune ne sont pas physiques car elles correspondraient à un écoulement avec une hauteur d'échelle inférieure à celle du plasma. De plus, les trajectoires de cette région ne pourraient être décrites que dans la portion où le nombre de Mach est inférieur à 1 (partie verte). En effet, elles se situent dans une région où la négativité est assurée partout et donc, si le nombre de Mach devenait plus grand que 1 , l'équation $V I-57$ nous permet d'affirmer qu'il devrait décroître (voir plus loin) et par conséquent stagner à $M_{2}=1$. Les courbes en rouge dans la région jaune pour $r<r_{c}$ correspondent à des écoulements physiques initialement supersoniques dont le nombre de Mach décroît dans la région où la friction domine. Ces écoulements ne sont donc pas adaptés au vent polaire qui est initié avec un nombre de Mach faible.

De ce que nous venons d'expliquer, il ressort essentiellement que seule la courbe en vert permet d'atteindre le point critique et d'assurer une transition d'un écoulement subsonique vers un écoulement supersonique. En effet au point critique, la courbe en vert vérifie $M_{2}=1$ et les termes sources se compensent exactement. Au-delà du point critique, la positivité est assurée car nous sommes dans la région de prédominance de l'accélération. Nous avons alors deux possibilités pour l'évolution du nombre de Mach de l'écoulement. Si le nombre de Mach immédiatement au-dessus du point critique est inférieur à 1 , ce que nous avons écrit nous permet d'affirmer que ce nombre décroît au-delà du point critique. C'est la courbe en vert qui sépare la région rose de la région jaune pour $r>r_{c}$. En fait cette solution n'est qu'asymptotique et correspond à une situation où le nombre de Mach est très voisin de 1 tout en restant inférieur à 1 . Les courbes en bleu de la région jaune pour $r>r_{c}$ correspondent à des écoulements subsoniques initiés dans la région où l'accélération domine et ne correspondent donc pas à des solutions adaptées au vent polaire. Si le nombre de Mach 1 est atteint au point critique et qu'il est supérieur à 1 juste au-dessus de ce point, alors l'évolution du nombre de Mach au-delà du point critique suit le signe du terme source, d'après VI-57 : si le terme source est positif, le nombre de Mach croît tandis qu'il décroît s'il est négatif. La variation est ainsi l'inverse de ce qu'elle est dans la région subsonique. Dans le cas de la tuyère de Laval, on retrouve cette particularité où la section droite $A$ de la tuyère joue le rôle de terme source : après franchissement du col de la tuyère, caractérisée par la section minimale, si le nombre de Mach est supérieur à 1 , il croît lorsque la tuyère s'évase, le point sonique étant atteint au col. 
La courbe en vert représente donc la seule solution qui permet d'aller d'un écoulement diffusif subsonique vers un écoulement supersonique. Les courbes en rouge dans la région verte correspondent à des solutions physiques où le nombre de Mach initial est plus grand que 1 et ne sont donc pas associées au vent polaire. De même la courbe en vert qui sépare la région jaune de la région verte pour $r<r_{c}$ n'est pas représentative du vent polaire.

En fin de compte, on peut associer au vent polaire deux familles de courbes :

- la famille de courbes de la région rose ; dans ce cas l'écoulement reste subsonique et tend vers 0 au fur et à mesure que l'on s'élève en altitude. On parlera alors plutôt d'une brise polaire ;

- la courbe en vert croissant avec l'altitude qui franchit le point critique. Cette courbe est la caractéristique du vent polaire. On peut noter que le point critique est à l'intersection des deux courbes en vert, solutions de l'équation VI-57. De ce point de vue, il constitue donc un point singulier pour cette équation qui peut être levé si on considère l'équation complète avec la dérivée temporelle.

Le mécanisme du vent polaire peut alors être résumé ainsi : au-dessus de la région de production de l'ion $\mathrm{H}^{+}$, le phénomène de diffusion dans l'ion majoritaire $\mathrm{O}^{+}$fait croître la vitesse de l'ion $\mathrm{H}^{+}$. A une altitude qui dépend fortement de la concentration de l'ion majoritaire $\mathrm{O}^{+}$, la vitesse est suffisamment grande pour que l'on atteigne $M_{2}=1$ et alors en ce point critique, la friction et l'accélération se compensent exactement. Au-delà de ce point, l'accélération due au champ de polarisation continue d'accélérer les ions qui peuvent ainsi atteindre puis dépasser la vitesse de libération, échappant ainsi à l'attraction gravitationnelle.

Il faut être conscient que nous décrivons le vent polaire thermique dans la région où l'approche fluide peut être considérée comme valable. De ce fait, le gain en énergie envisageable pour les ions est inférieur à $10 \mathrm{eV}$. Ce mécanisme d'extraction est en général relayé à haute altitude par des mécanismes qui peuvent apporter des gains énergétiques de plusieurs centaines d'eV, voire du $\mathrm{keV}$. Si on intègre ces mécanismes potentiels dans les sources de l'équation $V I-57$, les courbes en rouge qui se situent dans la région jaune pour $r>r_{c}$ correspondent à des solutions où les ions, initialement subsoniques (ils ont une courbe représentative dans la région rose), subissent une accélération brutale qui les placent dans des conditions d'échappement supersonique. De même les courbes en bleu de cette région jaune pour $r>r_{c}$, que nous avons dissociées du mécanisme du vent polaire, peuvent correspondre à une solution où l'échappement, initialement supersonique (courbe représentative en vert), devient brutalement subsonique (franchissement d'une onde de choc); ce phénomène peut correspondre à des situations où les conditions de pressions à l'infini ne sont brusquement plus compatibles avec un écoulement supersonique (phénomène transitoire de remplissage d'un tube de champ dans la plasmasphère après un sous-orage magnétique).

La description que nous venons de faire est toutefois un peu simplificatrice, en ce sens que nous n'avons considéré comme termes sources que l'accélération due au champ électrique de polarisation et la friction des ions $\mathrm{H}^{+}$dans les ions $\mathrm{O}^{+}$. En fait d'autres sources comme la diffusion thermique, très importante dans le cas de l'ion $\mathrm{H}^{+}$, ou le développement d'une anisotropie de pression de cet ion peuvent modifier les conditions de transition subsonique/supersonique, notamment en déplaçant l'altitude du point critique. Toutefois, ces 
sources supplémentaires ne remettent pas en cause le principe physique qui initie le vent polaire et que nous avons discuté.

La figure VI-21 ci-dessous présente les résultats d'une simulation de l'échappement des ions $\mathrm{H}^{+}$dans les conditions du vent polaire. Sur la figure de gauche, on a représenté le profil du nombre de Mach en identifiant la transition $M_{2}=1$. Sur la figure de droite, on a représenté les sources correspondant à l'accélération due au champ de polarisation et à la friction de l'ion $\mathrm{H}^{+}$dans l'ion $\mathrm{O}^{+}$(en fait l'opposé de cette friction) et on a identifié l'altitude où les deux termes se compensent. Nous constatons qu'il y a une différence d'altitude entre les deux points qui, dans le calcul que nous avons développé sont identiques. Cette différence provient du terme de diffusion thermique qui a pour effet principal de réduire la valeur effective de la fréquence de collision entre l'ion $\mathrm{H}^{+}$et l'ion $\mathrm{O}^{+}$d'un facteur proche de 2. La diffusion qui est associée est alors moins importante et le développement de la vitesse est plus faible en fonction de l'altitude ; il faut donc aller à plus haute altitude pour atteindre des vitesses qui permettent de franchir le point critique.

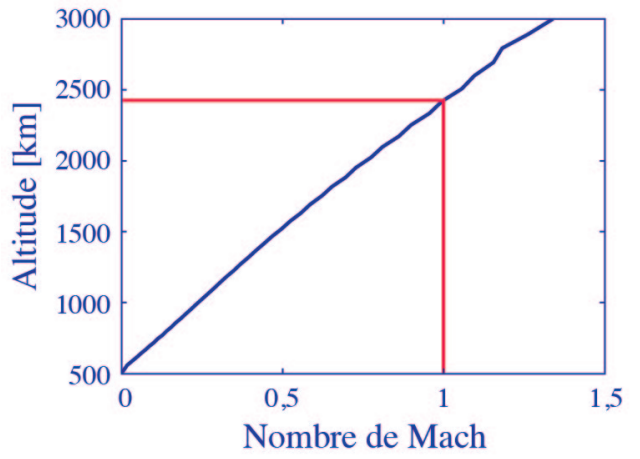

(a)

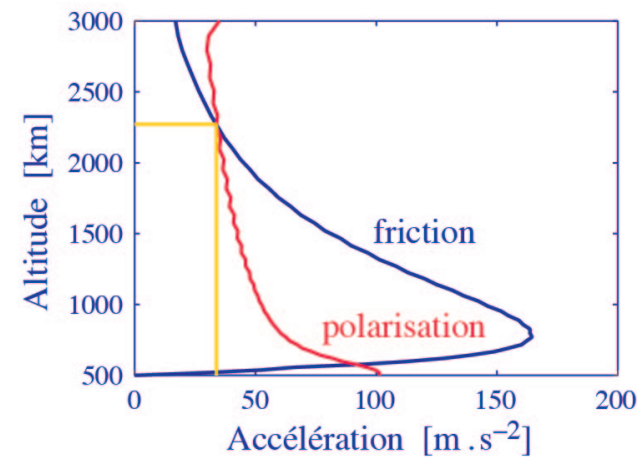

(b)

Figure VI-21 : profils verticaux pour l'ion $\mathrm{H}^{+}$, (a) du nombre de Mach et (b) des termes d'accélérations dus à la gravité et au champ de polarisation (courbe référencée polarisation) et à la friction de l'ion $\mathrm{H}^{+}$dans l'ion $\mathrm{O}^{+}$(courbe référencée friction)

En introduisant les effets d'anisotropie de pression, on trouve que le point critique correspond à une valeur de nombre de Mach supérieure à 1 (elle est proche de 1,1). Cette variation s'explique en partie par la définition que l'on adopte pour le nombre de Mach : on le définit avec une température supposée uniforme alors que l'anisotropie introduit des températures différentes suivant les directions.

La figure VI-22 représente la simulation de trois écoulements de l'ion $\mathrm{H}^{+}$. A gauche, on a représenté les profils de concentration des ions $\mathrm{H}^{+}$et $\mathrm{O}^{+}$correspondant à ces trois écoulements, et à droite, on a représenté le nombre de Mach de ces écoulements. La courbe en jaune correspond à l'équilibre hydrostatique et on note que le nombre de Mach correspondant est très faible. La courbe en bleu correspond à un écoulement diffusif dont le nombre de Mach reste subsonique et culmine vers $1800 \mathrm{~km}$. Enfin, la courbe en rouge correspond aux conditions d'échappement du vent polaire. On constate que lorsque le nombre de Mach 
reste subsonique, l'ion $\mathrm{H}^{+}$devient majoritaire à basse altitude et que l'altitude à laquelle il devient prépondérant dépend essentiellement du nombre de Mach maxima atteint (donc de la concentration de l'ion majoritaire) : plus ce nombre est élevé, plus l'altitude de transition entre $\mathrm{H}^{+}$et $\mathrm{O}^{+}$est élevée. Par contre, lorsque l'écoulement est supersonique, l'ion $\mathrm{H}^{+}$reste minoritaire jusqu'à très haute altitude, adoptant la hauteur d'échelle du plasma. La différence que l'on observe sur l'ion $\mathrm{O}^{+}$provient des conditions légèrement différentes utilisées pour le profil correspondant à l'échappement supersonique.

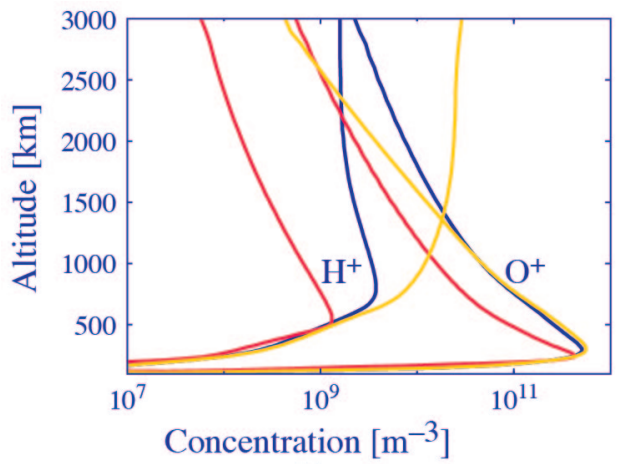

(a)

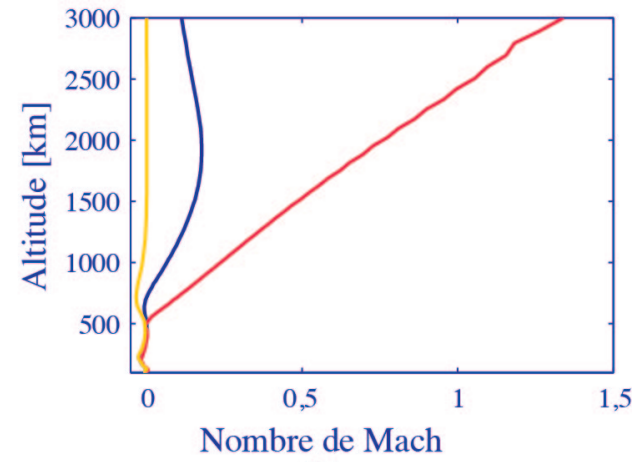

(b)

Figure $V I-22$ : profils verticaux (a) de la concentration des ions $\mathrm{O}^{+}$et $\mathrm{H}^{+}$ et (b) du nombre de Mach pour trois écoulements de l'ion $\mathrm{H}^{+}$: équilibre hydrostatique en jaune, équilibre diffusif en bleu et vent polaire en rouge

En fait, la caractéristique principale du vent polaire est que lorsqu'il existe et qu'il est stable, l'écoulement devient supersonique et l'ion $\mathrm{H}^{+}$est en échappement avec un flux limite imposé par la hauteur d'échelle de l'ion majoritaire $\mathrm{O}^{+}$. Dans la région d'écoulement subsonique, l'ion peut être considéré comme collisionnel alors qu'on peut le considérer non collisionnel dans la région d'échappement supersonique. Ces traits du vent polaire que nous avons particularisé en étudiant l'ion $\mathrm{H}^{+}$s'appliquent évidemment à tous les ions qui peuvent être accéléré comme l'ion $\mathrm{He}^{+}$, mais le phénomène est beaucoup moins marqué sur les autres espèces $\mathrm{du}$ fait de leur rapport de masse avec l'ion majoritaire $\mathrm{O}^{+}$. Cet échappement constitue une perte sèche d'ions $\mathrm{H}^{+}$et donc d'hydrogène atomique neutre. Cependant, la source d'hydrogène dans l'atmosphère est essentiellement le groupe hydroxyle $\mathrm{OH}$ et la molécule $\mathrm{H}_{2} \mathrm{O}$ qui ont un réservoir quasiment infini sur Terre ; cette perte est donc largement compensée.

\section{CALCUL DU VENT MÉRIDIEN : L'IONOSPHÈRE, TRACEUR DE LA THERMOSPHÈRE}

L'atmosphère n'est pas composée de deux parties indépendantes, l'une neutre et l'autre ionisée ; il existe entre la thermosphère et l'ionosphère, des couplages de divers ordres. Munis de la connaissance de la vitesse de diffusion des ions dans le gaz neutre, nous allons 
voir à présent qu'il est possible, à partir de la connaissance de paramètres purement ionosphériques, d'accéder à des données thermosphériques.

Les radars géophysiques dits «à diffusion incohérente » mesurent les températures ionique et électronique, la densité électronique, et la vitesse des ions le long de la ligne de visée (voir annexe III). Nous désirons étudier comment cette mesure permet d'accéder à des paramètres de l'atmosphère neutre. Pour cela, nous considérons le fait que le gaz neutre est beaucoup plus dense que le gaz ionisé et donc que la thermosphère impose ses mouvements à l'ionosphère à travers les collisions. Pour l'observateur au sol, le vent ionique observé le long de l'axe parallèle au champ magnétique est la somme du vent neutre plus la diffusion ambipolaire des ions dans les neutres, le tout projeté sur la ligne de visée. Pour des raisons qui apparaîtront clairement au cours du calcul, la ligne de visée sera considérée parallèle à la ligne locale du champ magnétique. La géométrie de ce problème est montrée sur la figure $V I-23$.

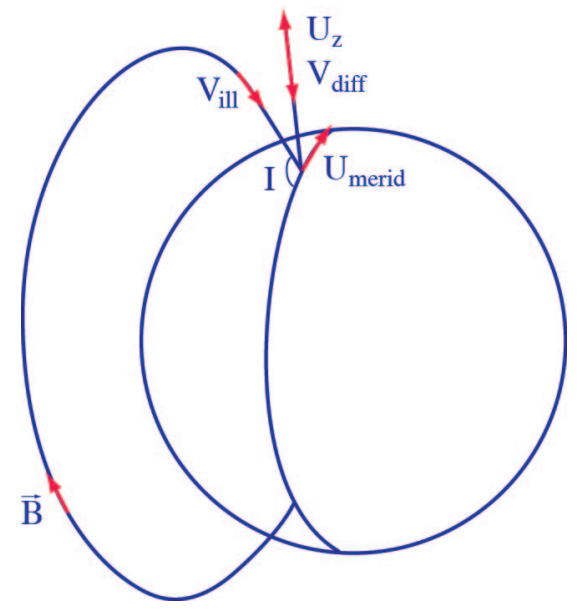

Figure VI-23 : coordonnées pour le calcul du vent méridien. I représente l'angle magnétique.

Sur cette figure, comme dans tout ce paragraphe, nous distinguons ce qui est vent ionosphérique (ionique) de ce qui est vent neutre de la façon suivante : $V$ ( $V$ majuscule) désignera une composante ionosphérique dans le repère de la Terre, et $U$ une composante thermosphérique. Les projections de la figure $V I-23$ conduisent à :

$V_{i / /}=U_{\text {merid }} \cos I+v_{\text {diff }} \sin I-U_{z} \sin I+U_{\text {zonal }} \cos \frac{\pi}{2}$

I est l'angle magnétique, c'est à dire l'angle entre la ligne de force du champ magnétique de l'horizontale. $U_{\text {merid }}$ est le vent méridien, c'est à dire le vent neutre le long d'un méridien géographique, positif vers le nord. $U_{z}$ est le vent vertical, positif vers le haut, nul en moyenne sur 24 heures, mais pouvant atteindre plusieurs dizaines de mètres par seconde en région $F$. $U_{\text {zonal }}$ est le vent est/ouest, positif vers l'est : sa projection sur la ligne de force du champ magnétique est nulle, ce qui signifie que les mesures par radar géophysiques ne permettent pas d'estimer cette composante du vent neutre. 
L'équation $V I-59$ peut se récrire :

$U_{\text {merid }}=-\frac{V_{\text {i/l }}}{\cos I}-v_{\text {diff }} \tan I+U_{z} \tan I$

$U_{z}$ n'est en général pas connu. Mais puisqu'il est nul en moyenne (par exemple sur 24 heures), on peut prendre la moyenne de l'équation $V I-60$ pour obtenir :

$<U_{\text {merid }}>=-\frac{<V_{i / /}>}{\cos I}-<v_{\text {diff }}>\tan I$

La vitesse de diffusion parallèle a été calculée au paragraphe $I V$ (équation $V I-47$ ). Elle dépend uniquement de la densité électronique, et des températures électroniques et ioniques. Ainsi, la mesure des paramètres de l'ionosphère permet de déduire les caractéristiques moyennes du vent méridien. Sur la figure $V I-24$, nous avons fait figurer à la fois la vitesse de diffusion et la vitesse parallèle, ainsi que le vent méridien déduit. Ces résultats correspondent à des mesures effectuées par le radar EISCAT lors de la même campagne d'expérimentation que celle utilisée pour le calcul de la vitesse de diffusion : 5 jours consécutifs en janvier 1993. Nous avons tracé ces vents à $250 \mathrm{~km}$ d'altitude. Nous pouvons voir que si la diffusion ne peut pas être négligée, elle joue cependant un rôle secondaire. Ainsi, le couplage dynamique se fait de la thermosphère vers l'ionosphère, le gaz neutre entraînant le gaz ionisé et non le contraire. L'ionosphère peut donc être considérée comme un traceur des vents thermosphériques.

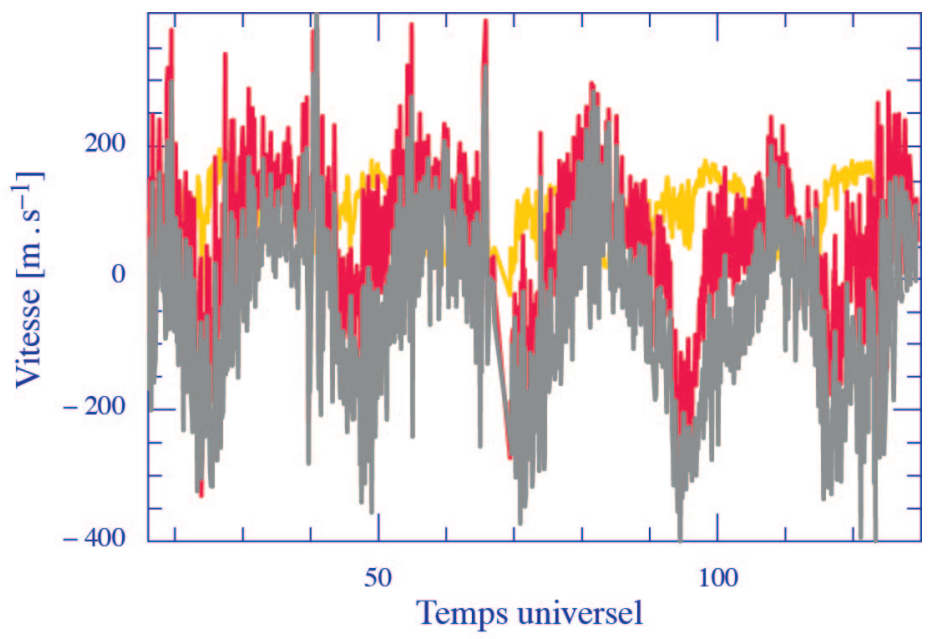

Figure $V I-24$ : la vitesse de diffusion est dessinée en jaune, la vitesse ionique projetée en rouge et le vent méridien en gris. Le temps est en heures.

Lors de ces jours calmes, nous avons des vitesses neutres qui varient typiquement de 200 à - $200 \mathrm{~m} \cdot \mathrm{s}^{-1}$ (soit $\pm 720 \mathrm{~km} / \mathrm{h}$ !). Pendant des jours agités, ces vitesses peuvent doubler. D'autre part, on distingue nettement des oscillations des vitesses neutres sur une échelle de quelques dizaines de minutes, avec des amplitudes de variation de plusieurs dizaines de mètres par seconde. 

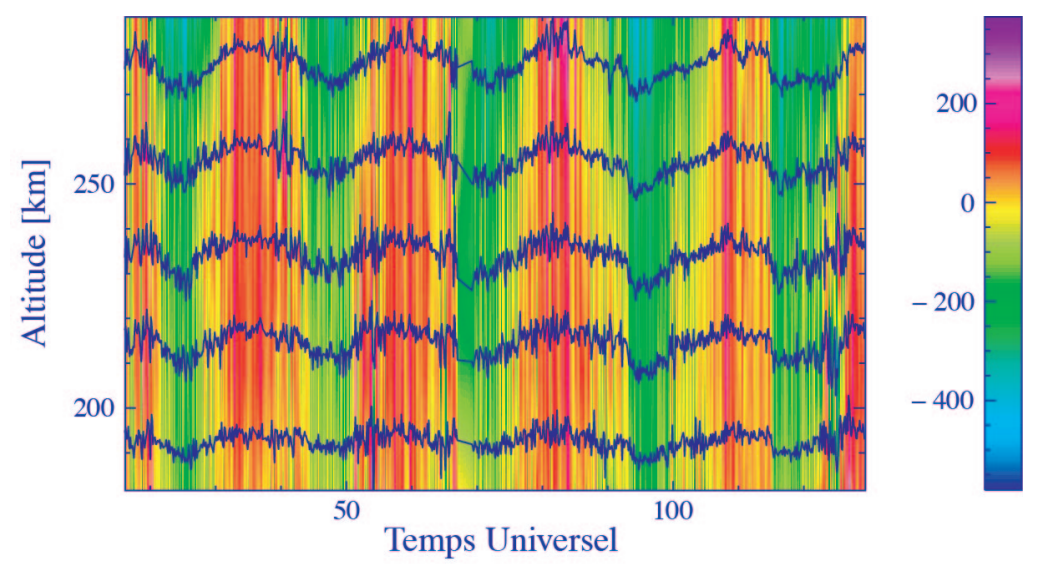

Figure VI-25 : vent méridien calculé à partir des mesures du radar EISCAT pendant cinq jours d'observations

Les heures sont exprimées en temps universel, à partir du premier jour d'expérience ( $25 \mathrm{TU}=$ une heure du matin le second jour). Il y a une heure d'avance du temps local sur le temps universel en Norvège, l'hiver. Un vent positif (rouge) va vers le nord. Les valeurs en rouge correspondent à un vent vers le nord de $200 \mathrm{~ms}^{-1}$ et les valeurs vertes à un vent vers le sud de $200 \mathrm{~ms}^{-1}$.

La figure VI-25 montre l'évolution du vent méridien à diverses altitudes. Le vent méridien évolue vers le nord autour de midi et vers le sud pendant la nuit. A partir des données radar, il est possible de connaître l'évolution du vent pendant tout un cycle solaire. C'est ce qui est représenté sur la figure $V I-26$.

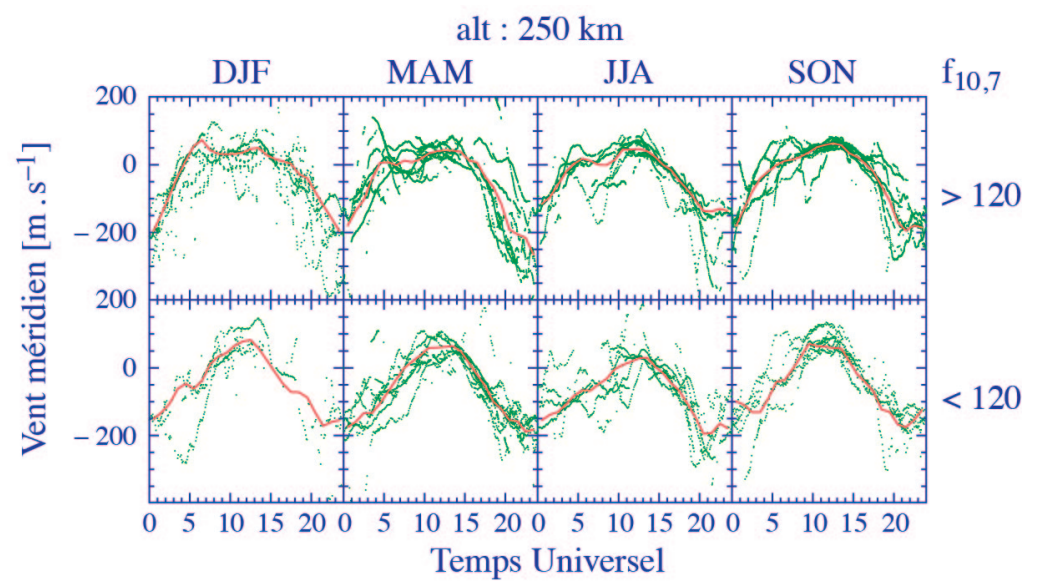

Figure VI-26 : évolution du vent méridien (positif vers le nord) en fonction de la saison et de l'activité solaire à haute latitude pendant un cycle solaire Les lettres DJF, MAM, JJA et SON correspondent aux premières lettres des mois de l'année. 
Ce dessin montre en points le vent méridien calculé à partir des données radar à haute latitude. La base de données comporte, à chaque altitude, 24948 points répartis sur onze années (soit un cycle solaire) allant de 1984 à 1995. Le trait rouge est une moyenne des points, qui montre l'évolution du vent. Nous ne montrons que l'altitude $250 \mathrm{~km}$, pour des fortes activités solaires en haut, et faibles en bas. Les conclusions sont que le maximum vers le nord est plus élevé en automne et hiver qu'au printemps ou en été. L'activité solaire importante retarde de plusieurs heures le passage du vent d'une direction sud à une direction nord. Enfin, lors d'activité faible, deux maxima vers le nord se dégagent en toute saison.

\section{DYNAMIQUE THERMOSPHÉRIQUE}

La dynamique grande échelle de la thermosphère a été présentée dans le chapitre $I I$. Dans ce paragraphe, nous continuons d'étudier l'ionosphère, traceur de la thermosphère, pour les marées et les ondes de gravité. Seule l'étude optique permet d'en connaître les tendances verticales ou dans la direction est/ouest, qu'on appelle zonale. Nous allons montrer ces tendances, et nous donnerons un aperçu du phénomène des marées thermosphériques.

\section{LES MARÉES THERMOSPHÉRIQUES}

Au chapitre II, nous avons vu qu'à l'encontre des marées océaniques, la source majeure des marées thermosphériques est l'échauffement diurne de l'ozone stratosphérique : elles trouvent donc leur origine au bas de la thermosphère, et non en haut, à travers l'absorption de l'ultraviolet proche par l'ozone stratosphérique et, dans une moindre mesure, l'absorption de l'infrarouge solaire par la vapeur d'eau troposphériques. Elles n'en sont pour autant pas plus simples que les marées océaniques. En effet, les gradients de température et de pression y jouent un rôle bien plus grand que dans la mer. L'absorption de l'énergie (en particulier du rayonnement solaire) par les différents composants de l'atmosphère tempère ou accentue ces gradients. Ainsi, les fréquences observées pour les marées thermosphériques ne se limitent pas à 24 et 12 heures (même si ce sont les deux plus importantes), et leurs phases varient avec l'altitude. La théorie mathématique des marées dépasse le cadre de cet ouvrage. Remarquons que la thermosphère étant un gaz, température, pression, vitesse, et densité y sont liées, et les marées peuvent y être étudiées sur n'importe laquelle de ces composantes. Le lien fort qui existe entre thermosphère et ionosphère fait qu'une fois encore, cette dernière peut servir de traceur à la première. La figure VI-27 montre les trois oscillations principales de ces marées sur les vitesses neutres parallèlement au champ magnétique, moyennées sur cinq jours de mesures avec le radar auroral EISCAT (12-16 février 1990). La vitesse neutre a été déduite de la mesure ionosphérique au moyen du développement du paragraphe précédent. On distingue les composantes diurne, semidiurne, dites aussi "solaires" car elles trouvent principalement leur origine dans l'éclairement solaire, et terdiurne, appelée aussi "lunaire". On voit que l'amplitude moyenne des marées est de $30 \mathrm{~m} \mathrm{~s}^{-1}$ à $230 \mathrm{~km}$ pour la composante diurne, de $12 \mathrm{~m} \mathrm{~s}^{-1}$ pour la composante 
semidiurne, et de $6 \mathrm{~m} \mathrm{~s}^{-1}$ pour la composante terdiurne. La variation de la température neutre due aux marées, à la même altitude, est de 40 à $100 \mathrm{~K}$ selon l'activité solaire.

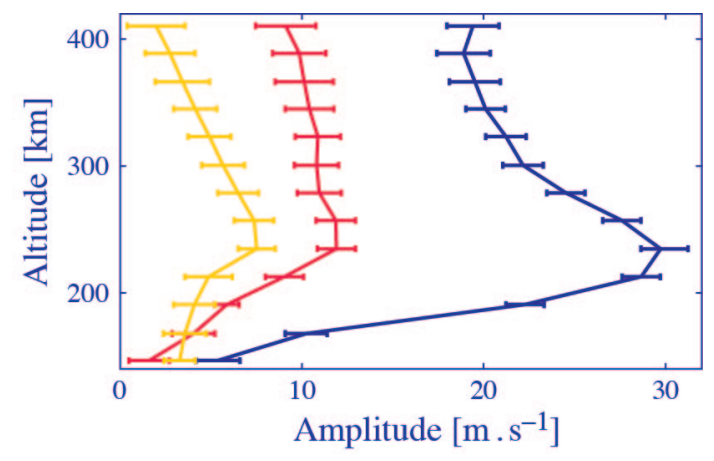

\section{Figure VI-27 : mesure des marées ionosphériques}

La composante diurne est en bleu, la composante semidiurne en rouge et la composante terdiurne en jaune.

De façon générale, les marées sont des oscillations périodiques qui affectent l'ensemble du globe. Dans la mésosphère, elles ont une amplitude typique de $50 \mathrm{~m} \cdot \mathrm{s}^{-1}$. Les composantes zonales et méridiennes y sont déphasées d'un quart de phase. La composante diurne est prépondérante à basses latitudes, tandis que la composante semi-diurne prédomine aux latitudes moyennes.

Ce n'est que récemment qu'on a pu mesurer les marées sur l'ensemble du globe. Sur la figure VI-28 nous montrons les vents méridiens mesurés par l'interféromètre WINDII (CNES/CSA/NASA) à bord du satellite d'observation de l'atmosphère terrestre UARS. Ces données sont déduites de la mesure de la raie $1 \mathrm{~S}$ de l'oxygène atomique et du rayonnement de $\mathrm{OH}$ dans la mésosphère et la basse thermosphère. L'équateur constitue une barrière pour ces vents. Dans cette région, l'atmosphère est divisée verticalement en régions convergentes et divergentes, selon la longueur d'onde de la marée (figure du haut). Pendant la période d'observation, (printemps 1992), la couche nocturne d'émission de O(1S) se trouve dans une région divergente, et possède donc un minimum d'intensité (figure du milieu), tandis que la couche d'émission de $\mathrm{OH}$, qui se trouve dans une région principalement convergente, possède un maximum à l'équateur (figure du bas).

\section{ONDES DE GRAVITÉ}

Le fait de considérer l'ionosphère comme traceur de la thermosphère a contribué à d'importantes avancées dans l'étude des ondes de gravité. Ces ondes font parties des diverses irrégularités de l'ionosphère, et sortent du cadre de ce livre, aussi n'en ferons-nous qu'une description sommaire. 

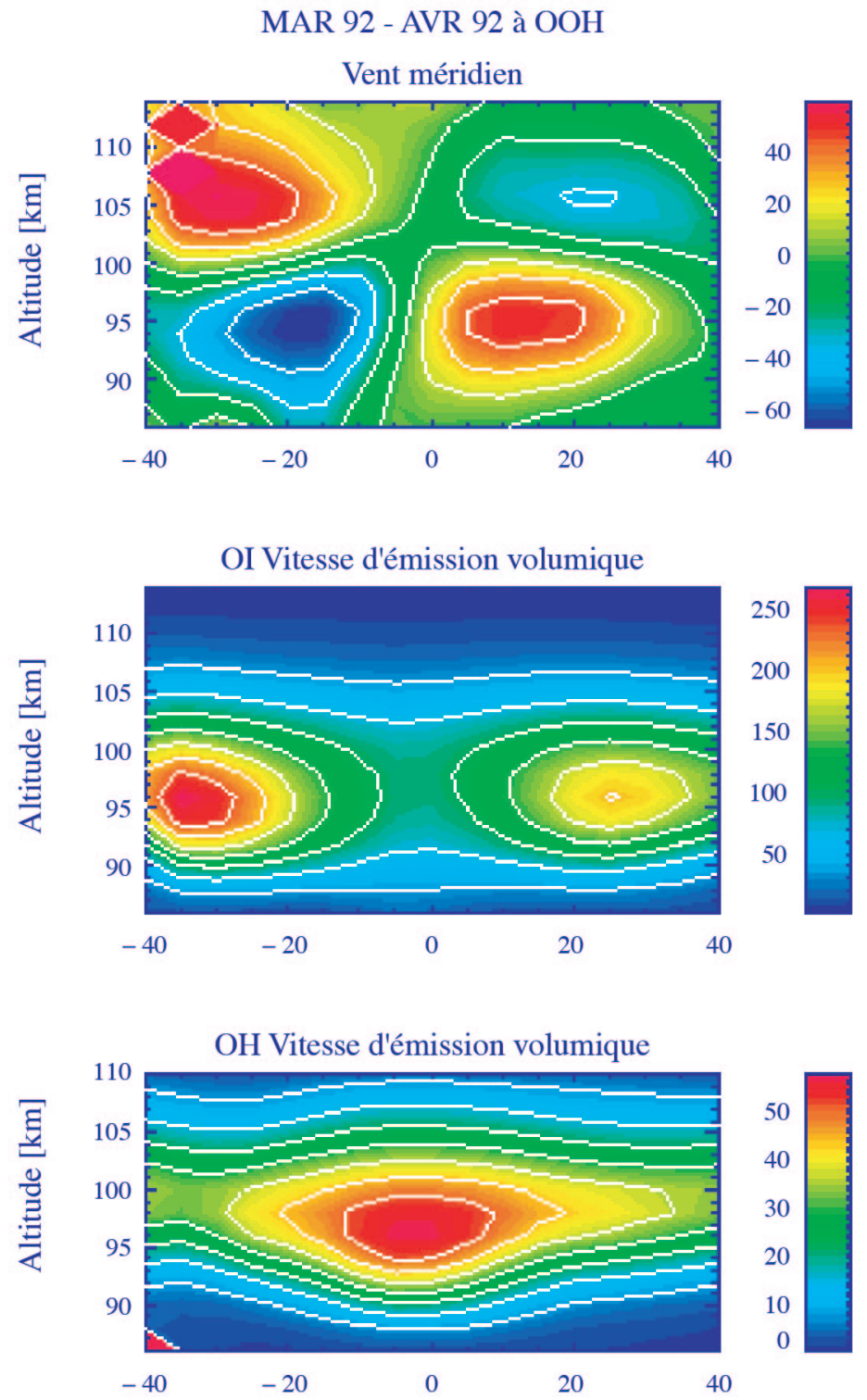

Figure $V I-28$ : mesure des marées ionosphériques par l'interféromètre WINDII à bord du satellite UARS

L'axe horizontal représente la latitude géographique (crédit NASA)

Dans les années soixante, diverses mesures révélaient que des ondes se propageaient dans la thermosphère, avec des variations de températures. Mais à l'inverse d'ondes acoustiques, leurs périodes relativement grandes - jusqu'à trois heures - et les longueurs d'onde en jeu, supérieures à $100 \mathrm{~km}$, font que dans leur modélisation, la gravité ne peut pas être négligée dans l'équation du mouvement face au gradient de pression. On nomma alors ces ondes ondes 
de gravité. D'autres perturbations, concernant cette fois l'ionosphère, existaient de la même sorte, se propageant avec des variations de densité, de vitesse et de température. Pour bien distinguer le phénomène thermosphérique et les effets ionosphériques, on appelle parfois ces derniers des perturbations ionosphériques mobiles. La relation entre les ondes de gravité et les perturbations ionosphériques a été mise en évidence seulement récemment : il fallut que des observations de types différents - et donc d'instruments différents et coordonnés montrent que ces deux types de perturbations sont corrélés. Puis il fallut que la modélisation explique cette corrélation.

Il s'agit d'ondes déclenchées par une perturbation locale dans la haute atmosphère, et qui se propagent vers d'autres latitudes : c'est le moyen de diffuser de grands apports d'énergie dans l'atmosphère. La perturbation peut être un champ électrique d'origine magnétosphérique brutalement augmenté, avec un chauffage Joule dans la région $E$, de précipitations intenses et locales, ou du « jet stream» des basses latitudes (voir chapitre $I I$ ). Cet apport violent d'énergie se dissipe dans l'atmosphère en générant des ondes matérielles. Comme les perturbations se situent le plus souvent à haute latitude, le parcours des ondes de gravité est le plus souvent du pôle vers l'équateur.

Que ce soient les ondes de gravité ou les perturbations ionosphériques mobiles, on distingue deux groupes, en fonction de la vitesse d'onde et de la période.

Les ondes de grande échelle se propagent dans la thermosphère et ont des vitesses verticales comprises entre 400 et $1000 \mathrm{~m} \mathrm{~s}^{-1}$, c'est-à-dire de l'ordre de la vitesse du son dans la thermosphère. Leur longueur d'onde horizontale est supérieure à $1000 \mathrm{~km}$, et leur période va de la demi-heure à trois heures.

Les ondes de moyenne échelle possèdent des vitesses verticales de l'ordre de 100 à $250 \mathrm{~m} \mathrm{~s}^{-1}$, c'est à dire inférieures à la vitesse du son, des longueurs d'onde de quelques centaines de km, et des périodes allant de la demi-heure à l'heure. Sur la figure VI-29, des ondes de moyenne échelle sont mises en évidence lors de mesures ionosphériques (il s'agit alors de «perturbations ionosphériques mobiles»). Sur cette figure, une analyse temps fréquence a été mise en œuvre sur les données de vitesse ionique acquises par le radar EISCAT, lors d'une campagne le 24 mars 1995.

Cette expérience dont la figure $V I-18$ représente l'évolution temporelle des principaux paramètres du plasmas mesurés par le radar commence après $14 \mathrm{TU}$ (soit 15 heures locales), et dure 11 heures. Les indices planétaires ne témoignent guère de l'activité qui règne ce jour là : l'indice $\mathrm{f}_{10.7}$ vaut 100 , avec une moyenne à 3 mois de 81,6 , et l'indice $\mathrm{Ap}$ vaut 5 . Localement cependant, des périodes de fort champ électrique alternent avec des périodes calmes, et avec des précipitations de particules. L'analyse de Fourier de la vitesse ionique (dont nous savons à présent qu'elle est traceur de la vitesse thermosphérique) montre clairement qu'une onde se propage entre le début de l'expérience et environ 16:30 TU. Sa période est de l'ordre de la demi-heure. Un simple regard à la vitesse ionique elle-même suffit à mettre cette onde en évidence (l'analyse mathématique ne fait que la confirmer), comme nous le montrons dans le cadre du bas. Les perturbations visibles à toutes les fréquences entre 17:00 TU et 20:00 TU n'ont probablement pas de signification 
physique : en effet, cela correspond au coucher du Soleil. L'ionosphère se vide alors, et le radar est en limite de sa sensibilité de mesure.

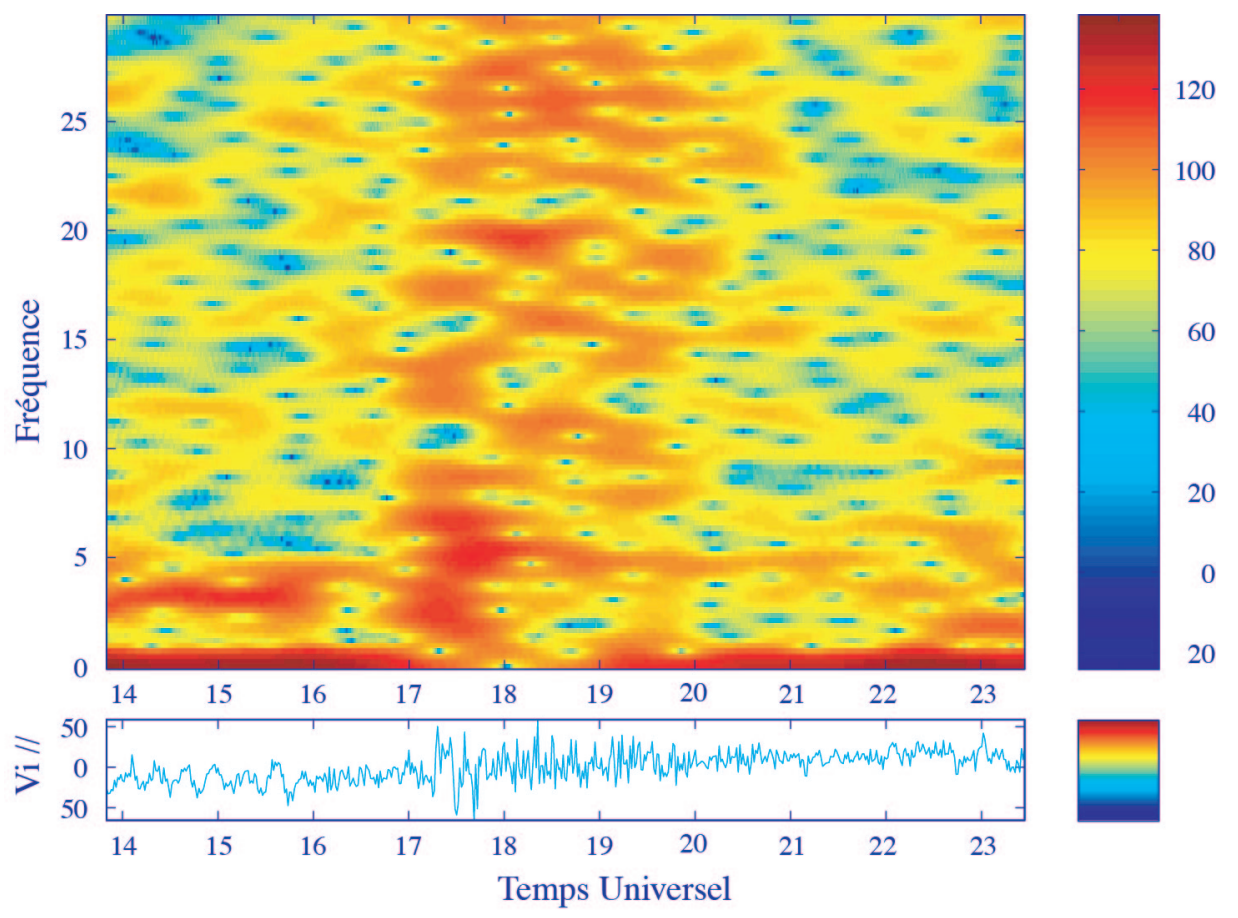

Figure VI-29 : analyse temps-fréquence de données de vitesse ionique

Sur la figure du haut, nous avons fait figurer en ordonnée la fréquence, exprimée en heure ${ }^{-1}$, et le temps universel en heure est en abscisse. Sur la figure du bas, nous montrons la vitesse ionique elle-même en fonction du temps.

La figure $V I-30$ distingue quatre modes de génération des ondes de gravité. Ils trouvent leur origine dans une perturbation de la basse thermosphère.

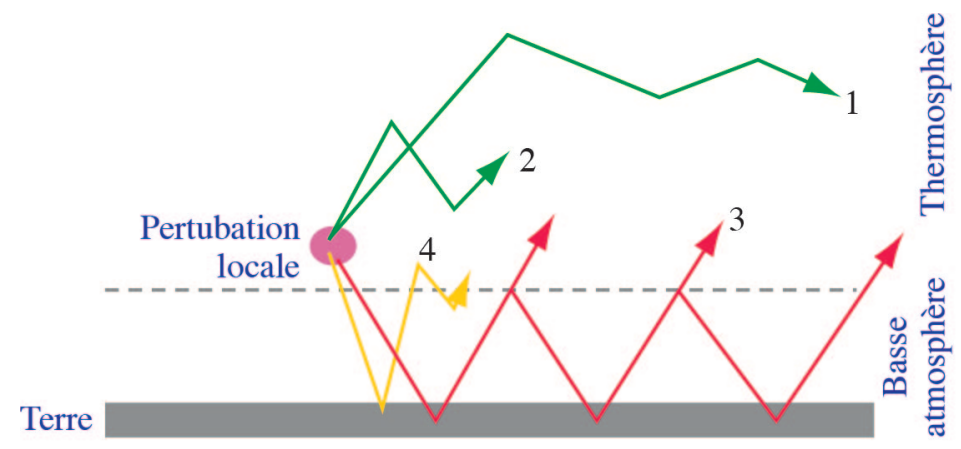

Figure VI-30 : les quatre modes de propagation des ondes de gravités

(d'après Mayr et al., 1990) 
Les modes vers le haut (modes 1 et 2 ) sont des modes directs, qui se propagent quasiment horizontalement dans la thermosphère. Le mode direct est piégé par le gradient de température et domine à grande distance de la source. A moyenne latitude, on l'observe se propageant vers l'équateur. De même nature, un mode de plus courte longueur d'onde, se propage dans un cône proche de la source. Ces deux modes sont des modes de grande échelle. Les modes de moyenne échelle commencent leur propagation vers le bas de la source (modes 3 et 4 ). Ils sont piégés par les réflexions sur la surface terrestre ou par les gradients thermiques, et se propagent des hautes latitudes vers les moyennes.

La figure VI-31 montre une mesure spectaculaire de perturbations ionosphériques mobiles. Elles ont été acquises par un des radars cohérents de la chaîne de radars SuperDarn (voir annexe III). Les ondes de ces radars ne sont pas diffusées par l'ionosphère comme dans le cas de la diffusion incohérente, dont nous avons présenté de nombreux exemples. Ici, le faisceau se réfléchit dans la région $F$. L'altitude de réflexion dépend de la concentration électronique, traceur encore une fois de la thermosphère. Une perturbation mobile qui se propage change l'altitude de réflexion de l'onde radar, et en permet ainsi la visualisation. La figure VI-31 a été acquise avec le radar de Goose Bay le 5 janvier 1995 (Bristow et Greenwald, 1997). L'expérience a duré de 12:00 TU à 22:00 TU.
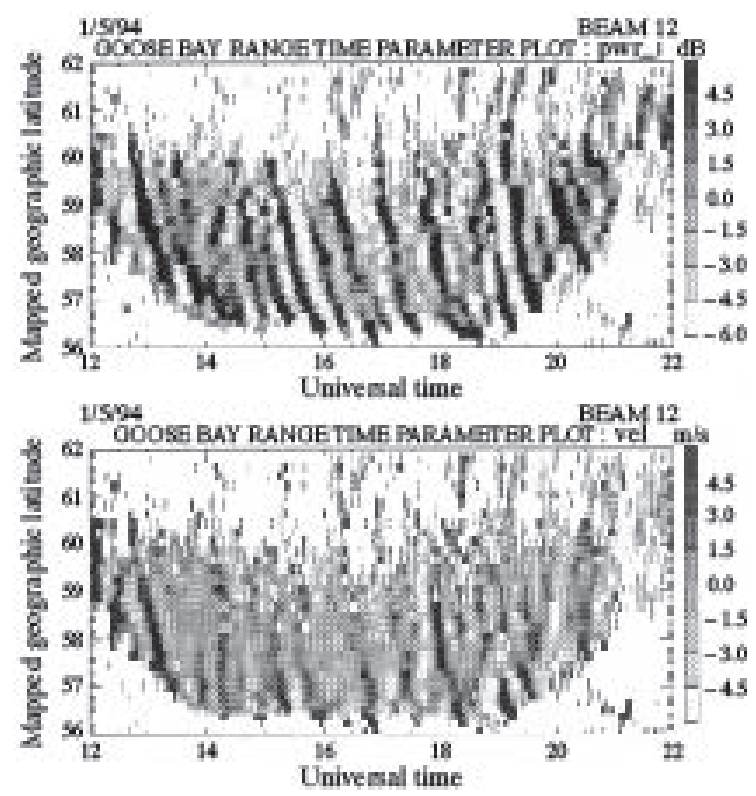

Figure VI-31 : mesure des ondes de gravité par le radar à diffusion cohérente de Goose Bay

En haut, puissance de la perturbation $(\mathrm{dB})$, et en bas, vitesse $\left(\mathrm{m} \cdot \mathrm{s}^{-1}\right)$. Les données ont été filtrées pour faire ressortir les caractéristiques des périodes entre 15 et 90 minutes (Bristow et Greenwald, J. Geoph. Res., 102, 11585-11595, 1997) 
Les données montrent clairement des ondes de gravité, quasi périodiques, qui se propagent du nord vers le sud. Ces ondes sont plus visibles sur la puissance de la perturbation (figure du haut). Leur période est de l'ordre de la demi-heure. Un effet des marées ionosphériques est de transporter les particules ionisées des hautes latitudes vers les basses latitudes. C'est ainsi que même en période calme, en l'absence de toute précipitation en dessous de $60^{\circ}$ de latitude, il existe une ionosphère nocturne équatoriale. Sa région $F$ montre des ondulations de période typique de deux heures, en accord avec les caractéristiques d'ondes de gravité à grande échelle : l'altitude du pic monte et descend de plusieurs dizaines de kilomètres. Il se développe aussi une région $E$ aux latitudes équatoriales, dont l'origine est encore un transport depuis les latitudes plus élevées. Des perturbations aux hautes latitudes conduisent parfois à gonfler la région $F$ qui, au gré des marées, semble se vider vers la région $E$. Ce phénomène sur lequel nous ne nous étendrons pas a reçu le nom de «couche $E$ sporadique ».

\section{RÉSUMÉ}

Ce chapitre fait le lien entre diverses parties de l'environnement terrestre étudiées précédemment : magnétosphère, thermosphère, ionosphère.

La magnétosphère est liée à l'ionosphère de deux façons : particulaire et électromagnétique. Les particules sont les électrons et protons du vent solaire qui précipitent dans l'ovale auroral ou la calotte polaire. Leur spectre énergétique va de la centaine d'électronvolts à quelques dizaines de kilo-électronvolts. Le couplage électromagnétique se fait à travers les courants de Birkeland, ou l'électrojet auroral, qui bouclent dans l'ionosphère vers $110 \mathrm{~km}$, région appelée couche de conductivité perpendiculaire élevée. Les courants induits sont le courant de Hall (courant perpendiculaire à la fois au champ électrique et au champ magnétique) et le courant de Pedersen (perpendiculaire au seul champ magnétique). A ces courants correspondent des conductivités élevées, appelées conductivités de Hall et Pedersen, qui sont principalement fonction de la densité électronique.

Aux hautes latitudes, le plasma ionosphérique est entraîné dans un mouvement de convection suivant un schéma type de deux cellules contrarotatoires, par le champ électrique présent dans l'ionosphère, qui résulte de la projection le long des lignes de champ (qui sont des équipotentielles) du champ électrique existant dans le plan équatorial de la magnétosphère.

Cette convection a pour effet de créer un mouvement différentiel entre les ions et les composants neutres de l'atmosphère et de provoquer l'apparition de forces de friction. Ces forces de friction sont à l'origine de la dissipation dans l'ionosphère, sous la forme d'effet Joule, de l'énergie magnétosphérique transmise au plasma ionosphérique et résulte en un échauffement immédiat du plasma et à terme de la thermosphère. Cet échauffement, qui peut être important (un accroissement de 1500 à $2000 \mathrm{~K}$ n'est pas rare) affecte considérablement la chimie de l'ionosphère et en particulier l'équilibre ions atomiques / ions moléculaire. Par ailleurs, cette friction est source d'anisotropie pour ce plasma avec une température perpendiculaire aux lignes de champ supérieure à la température parallèle, qui peut 
avoir comme conséquence de modifier sensiblement les fonctions de distribution des ions, les rendant fortement non maxwelliennes.

Enfin, les hautes latitudes sont également caractérisées par des lignes de champ fortement étirées, voire ouvertes et reconnectées, qui sont propices à l'apparition du vent polaire, un mécanisme d'extraction des ions légers depuis l'ionosphère. Les ions du vent polaire sont créés dans l'ionosphère puis sont accélérés par le champ électrique de séparation de charge, qui compense largement l'action de la gravité. Les vitesses acquises sont très importantes et l'écoulement est de nature supersonique.

L'ionosphère est plongée dans la thermosphère. Il existe entre ces deux parties de l'atmosphère un couplage dont témoignent bien les fréquences de collisions étudiées au chapitre $V$. En raison de ces collisions importantes, le vent neutre et le vent ionique sont liés, mais nous avons montré que l'entraînement se fait de la thermosphère vers l'ionosphère. L'énergie considérable déposée aux pôles se dissipe en partie en engendrant des mouvements du système couplé thermosphère/ionosphère vers l'équateur. Ces mouvements de grande amplitude sont périodiques de périodes comprises entre le quart d'heure et l'heure. On les appelle ondes de gravité. Les marées ionosphériques, de périodes plus élevées, trouvent quant à elle leur origine dans l'énergie déposée dans la très basse thermosphère par les phénomènes géophysiques réguliers (rotation de la Terre).

\section{BIBLIOGRAPHIE}

Un ouvrage en français, déjà cité, traite bien de la dynamique ionosphérique. Il s'agit de :

Physique de l'ionosphère, de A. Giraud et M. Petit, aux Presses Universitaires de France, paru en 1975.

Les deux articles de base sur les marées sont :

J.M. Forbes, Atmospheric tides 1. Model description and results for the solar diurnal component, J. Atmos. Terr. Phys., 87, 5222-5240, 1982.

J.M. Forbes, Atmospheric tides 2. The solar and lunar semidiurnal component, J. Atmos. Terr. Phys., 87, 5241-5252, 1982.

Même si ces articles ne sont plus récents, ils font encore référence sur le sujet. Cependant, un article extrêmement pédagogique et rigoureux, avec de nombreuses références (en particulier historiques) mérite d'être cité :

Atmospheric tides in the mesosphere and lower thermosphere of the Earth, G. Thuiller and F. Vial, ERCA 3, EDP Sciences, Paris, IBSN 2-86883-389-6, 1999.

Un auteur s'est particulièrement penché sur la dynamique et l'électrodynamique ionosphérique. Il a écrit un livre, qui traite entre autres de toutes les irrégularités, dont les ondes de gravité. Il s'agit de :

The Earth's Ionosphere, Plasma physics and electrodynamics, M.C. Kelley, Academic Press Inc., ISBN 0-12-404013-6, 1989. 
La relation entre les ondes de gravité et les perturbations ionosphériques a été mise en évidence seulement récemment. On lira en particulier :

Kirchengast, G., Elucidation of the physics of the gravity wave - TID relationship with the aid of theoretical simulations, J. Geophys. Res., 101, 13353-13368, 1996.

Le vent neutre a été étudié au moyen d'instruments optiques dans la région aurorale par :

Fauliot, V., G. Thuillier, and M. Hersé, Observations of the F-region horizontal and vertical winds in the auroral zone, Ann. geophysica, 11, 17-28, 1993. 
